\title{
(2) \\ Modeling of Multicomponent Diffusions and Natural Convection in Unfractured and Fractured Media by Discontinuous Galerkin and Mixed Methods
}

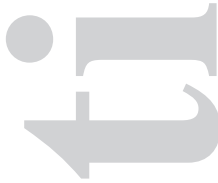

${ }^{1}$ Physical Science and Engineering Division, King Abdullah University of Science and Technology, Thuwal, Kingdom of Saudi Arabia,

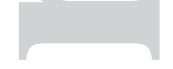

\author{
${ }^{2}$ RERI and Yale University, Palo Alto, California, USA
}

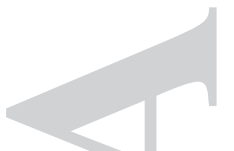

\section{SUMMARY}

Computation of the distribution of species in hydrocarbon reservoirs from diffusions (thermal, molecular, and pressure) and natural convection is an important step in reservoir initialization. Current methods, which are mainly based on the conventional finite difference approach, may not be numerically efficient in fractured and other media with complex heterogeneities. In this work, the discontinuous Galerkin (DG) method combined with the mixed finite element (MFE) method is used for the calculation of compositional variation in fractured hydrocarbon reservoirs. The use of unstructured gridding allows efficient computations for fractured media when the crossflow equilibrium concept is invoked. The DG method has less numerical dispersion than the upwind finite difference (FD) methods. The MFE method ensures continuity of fluxes at the interface of the grid elements. We also use the local discontinuous Galerkin (LDG) method instead of the MFE calculate the diffusion fluxes. Results from several numerical examples are presented to demonstrate the efficiency, robustness, and accuracy of the model. Various features of convection and diffusion in homogeneous, layered, and fractured media are also discussed. Copyright (c) 2017 John Wiley \& Sons, Ltd.

\section{Received ..}

KEY WORDS: Compositional modeling, fractured porous media, discrete fracture model, convection-

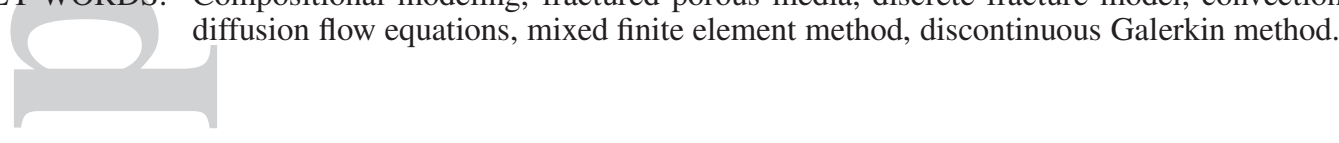

\section{INTRODUCTION}

Many reservoirs around the world exhibit compositional variations, vertically and laterally [1, 2, 3]. These gradients can significantly affect the hydrocarbon characteristics such as the API gravity, producing gas-oil-ratio (GOR), saturation pressure and the total hydrocarbons in place. Reservoir fluid initialization has a major impact on reservoir production predictions. The number and location of offshore wells are critically related to the distribution of species in the subsurface formation. For instance, Metcalfe et al. [4] reported significant compositional gradients in the Anschutz Ranch East field which resulted in different GOR behaviors observed at different wells in the

${ }^{*}$ Correspondence to: Hussein Hoteit, Physical Science and Engineering Division, King Abdullah University of Science and Technology,Thuwal 23955-6900, Kingdom of Saudi Arabia Email: hussein.hoteit@kaust.edu.sa

This article has been accepted for publication and undergone full peer review but has not been through the copyediting, typesetting, pagination and proofreading process, which may lead to differences between this version and the Version of Record. Please cite this article as doi: $10.1002 / \mathrm{nme} .5753$ 
field. In a big reservoir (43km long, $23 \mathrm{~km}$ wide) in the Middle East, Hamoodi at al. [5] observed large compositional variations in the areal extent. Some components showed 10-fold difference in composition across the reservoir. Similar phenomena have also been observed in many other fields $[6,7,8,9,10]$. There are three distinct processes which affect species distribution in hydrocarbon reservoirs. These include: 1) natural convection, 2) different diffusion mechanisms, and 3) reservoir filling and leakage [11]. Natural convection is mainly affected by horizontal temperature gradient. Diffusions are from temperature, pressure, and composition gradients. Reservoir filling and leakage are from the sources outside of the reservoir domain. Current practice in reservoir engineering is mainly based on one-dimensional vertical variation in composition with depth due to gravity and thermal gradients $[12,13]$. Horizontal species distribution is often ignored in the reservoir. When there is no reservoir filling and leakage, compositional variations in connected hydrocarbon reservoirs are mainly from natural convection, and Fickian, pressure, and thermal diffusions. There are only a few studies in the literature that incorporate the effect of diffusions and natural convection on hydrocarbon species distribution. Jacqmin [14] investigated the compositional variation in homogeneous porous media in accounting for natural convection and Fickian diffusion in a binary mixture. Riley and Firoozabadi [15] studied the effect of the thermal, pressure, and Fickian diffusion as well as natural convection for a single-phase, two-component fluid in a homogeneous crosssectional reservoir with a given linear temperature distribution. For a methane $\left(C_{1} /\right.$ normal $)$ butane $\left(n C_{4}\right)$ binary mixture, they reported that methane segregates towards the bottom-hot side of the domain. They also showed that a small amount of convection could cause the horizontal composition gradient to increase until a certain limit which varies with permeability. They solved the steady state mass conservation equation and Darcy's law, using a successive iteration approach based on Poisson's equation[15]. This method is accurate in approximating the horizontal compositional variation. However, the convergence rate is slow and therefore it is computationally costly at high permeabilities. Ghorayeb and Firoozabadi $[16,17]$ used the FD method to investigate the diffusion and convection effects in multicomponent mixtures in homogeneous 2D rectangular domains. In their implementation, the steady state solution was obtained by solving the unsteady state equations and iterating on time until reaching convergence. They found that, unlike in binary hydrocarbons, methane in ternary or multicomponent mixtures can get to a higher concentration at the cold top-side in a rectangular domain. Later, Ghorayeb and Firoozabadi [18] used the same numerical approach to approximate the multicomponent diffusion and convection in a fractured rectangular domain. A uniform network of connected and disconnected fractures was considered using a single-porosity model. Because of the length-scale and permeability contrasts between the matrix and the fracture grid cells, the numerical discretization gave rise to large and ill-conditioned linear systems. For a $30 \mathrm{~m}$ by $15 \mathrm{~m}$ fractured cross-section with a $1-\mathrm{mm}$ fracture aperture, a gird of about 45,000 elements was required. Consequently, this approach may not be practical for compositional variation calculations in fractured reservoirs of more realistic geometrical dimensions.

Ghorayeb and Firoozabadi $[16,17,18]$ assumed that the Oberbeck-Boussinesq approximation was valid for a limited range of temperature, pressure, and composition. In this work, we consider the unsteady state convection-diffusion flow equations without Oberbeck-Boussinesq assumption. The fluid density is calculated using the Peng-Robenson equation of state (PR-EOS). Our requirements for the numerical solution of the flow equations are: 1) mass is conserved locally at the cell level, 2) numerical dispersion is low enough to study physical dispersion/diffusion, and 3) unstructured grids are used to provide flexibility to describe complicated geometries including fractures. In previous studies[19, 20, 21], we provided a detailed description of the numerical approach in approximating pure convective multicomponent flow equations in fractured media. The discontinuous Galerkin method was used to solve the convection-diffusion equations. The FD method has poor accuracy on unstructured girds and lacks the flexibility to describe complicated geometries. Our objective is to develop an accurate and efficient numerical method for calculating the compositional variation of multicomponent mixtures, taking into account the diffusions and natural convection processes in homogeneous and fractured media with connected and disconnected fractures.

This article is protected by copyright. All rights reserved. 
Numerical approximations based on cell-centered finite volume methods compute the surface integrals (convection and diffusion fluxes) at the cell boundaries. Russell and Wheeler [22] showed that the MFE method, with a surface integration formula, becomes equivalent to the FD method on structured grids (see also [23]). The formulation was used by Weiser and Wheeler[24] to develop optimal convergence for both pressure and velocity in cell-centered FD methods in rectangular grids. Arbogast et al. [25, 26] extended this formulation to full tensors in triangular and logically rectangular grids. In distorted quadrilaterals and hexahedra, a multipoint flux mixed finite element method was later developed by Wheeler et al. [27, 28]. For unstructured grids, the MFE method is superior to the conventional finite element and finite volume methods in terms of flux accuracy, particularly with a strong discontinuity in the transmissivity [29, 30]. In this study, we use the MFE method with the lowest order Raviart-Thomas space [31]. The main features of the MFE method are: 1) the pressure and fluxes are approximated simultaneously with the same order of convergence; 2) a locally conservative method suitable to model full permeability tensors. The MFE method may produce ill-conditioned linear systems where the cell pressure averages and the inter-element fluxes are the primary unknowns. To overcome this issue, we use the hybridized MFE technique $[32,33,34]$ where the primary unknowns are the edge pressure averages (pressure traces). In this paper, we refer to the hybridized MFE method by the MFE method.

With natural convection, the magnitude of the flow velocity is relatively very small (in the order of $10^{-10} \mathrm{~m} / \mathrm{s}$ ) compared to the case when the field is under primary or secondary depletion. We found that high accuracy in approximating the convective fluxes is needed to capture the convective fluid circulations in the reservoir. We provide a numerical example showing that the calculation of natural convective velocity by the standard two-point FD approximation leads to inaccuracy in the velocity field in heterogeneous media. Therefore, we recommend using the MFE method. One drawback of the MFE method in calculating the diffusion fluxes is the size of the linear system which is equal to the number of the mesh edges. To overcome this difficulty, an alternative approach is to use the local discontinuous Galerkin (LDG) method. Other authors coupled the DG and MFE methods $[35,36,37]$ for similar applications.

This work provides an original contribution to the numerical modeling of muticomponent fluid flow in fractured media in the context of natural convection and diffusions. The main new contributions can be summarized in two aspects: 1) we investigated the impact of reservoir heterogeneity and fractures with unstructured distribution on the fluid flow circulation and hydrocarbon compositional variation in a reservoir from natural convection and diffusions. 2) we proposed a numerical approach that combines the MFE and LDG methods to solve the convectiondiffusion equations, where diffusions are described by a full diffusion tensor that is a function of pressure, temperature and composition.

The LDG method was first introduced by Cockburn and Shu [38] as an extension of the DG method to approximate the convection-diffusion equation. This method is similar to the MFE method concerning the flux calculation; the primary unknown and its gradient are approximated simultaneously. Unlike the MFE method, the LDG method allows a local evaluation of the diffusion fluxes. However, we demonstrate that the MFE method is more accurate in approximating the diffusion fluxes in non-smooth finite element grids.

In this study, we use the concept of crossflow equilibrium to represent flow in fractured media. The model based on this concept has been referred to as 'the discrete fracture model' and is an alternative to the single- or dual-porosity model. It can be used to describe connected and disconnected fractures. Because the fracture aperture is very small compared to the matrix size, this approach allows us to represent the fractures using $1 \mathrm{D}$ linear elements within a $2 \mathrm{D}$ system $[39,40]$. Thus, a fracture entity can be logically represented by the cell edges. This simplification of the fractures reduces significantly the computational requirements. We use the MFE method to approximate the pressure and the velocity in the fractures. As a result, the fluxes at the intersection of multi-fractures are correctly calculated. In this paper, we first present a mathematical model describing the convection-diffusion flow of multi-component fluids; we then briefly describe the numerical model that combines the MFE and DG methods. Convection and diffusion fluxes are approximated by both the MFE and LDG methods. We present numerical examples comparing the 
MFE and the two-point FD methods for the convection fluxes with the MFE and LDG methods for the diffusion fluxes. To demonstrate the efficiency and robustness of our numerical approach, we provide examples that cover homogeneous, heterogeneous and fractured media.

\section{MATHEMATICAL MODEL}

We consider a single-phase system with $n_{c}$ components. For each component $i$, the mass balance is expressed by the following convection-diffusion equation:

$$
\phi \frac{\partial z_{i} c}{\partial t}+\nabla \cdot\left(z_{i} c \boldsymbol{\vartheta}\right)+\nabla . \boldsymbol{J}_{i}=0 \quad i=1, \ldots, n_{c} \quad \text { in } \quad \Omega \times(0, \tau),
$$

where $\phi$ is the porosity, $c$ is the molar density, $z_{i}$ is the mole fraction of component $i, \boldsymbol{J}_{i}$ is the molar diffusion flux of component $i, \Omega$ is the $2 \mathrm{D}$ computational domain, and $\tau$ denotes the simulation time.

The bulk velocity field $\vartheta$ is given by Darcy's law:

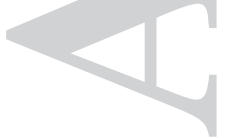

$$
\vartheta=-\frac{\boldsymbol{k}}{\mu}(\nabla p-\rho \boldsymbol{g}) \quad \text { in } \quad \Omega \times(0, \tau),
$$

where $k$ is the absolute permeability tensor, $\mu, p, \rho$ are the viscosity, pressure, and mass density, respectively. The gravitational constant vector $g$ is oriented downward.

The flow equations 1 and 2 are coupled with the Peng-Robenson EOS that models the density as a function of composition, temperature, and pressure:

$$
\left\{\begin{array}{l}
\rho=c M \\
c=\frac{p}{Z R T} \\
Z^{3}-(1-B) Z^{2}+\left(A-3 B^{2}-2 B\right) Z-\left(A B-B^{2}-B^{3}\right)=0
\end{array}\right.
$$

where $M$ is the molecular weight, $R$ the gas constant, $T$ the temperature, $A$ and $B$ the PR-EOS parameters [41, 42].

Ghorayeb and Firoozabadi [43] expressed the diffusion mass flux $\boldsymbol{J}_{i}$ in a single phase mixture in the general form:

$$
\boldsymbol{J}_{i}=-c\left(\sum_{j=1}^{n_{c}-1} D_{i j}^{M} \nabla z_{j}+D_{i}^{p} \nabla p+D_{i}^{T} \nabla T\right)
$$

where $D_{i j}^{M}, D_{i}^{p}$, and $D_{i}^{T}$ are, respectively, the coefficients of the molecular diffusion between components $i$ and $j$, pressure diffusion, and thermal diffusion. Details of the diffusion coefficients are provided in references [43, 44].

Summing up Eq. 1 over all components and using the facts that $\sum_{i=1}^{n_{c}} z_{i}=1$, and $\sum_{i=1}^{n_{c}} \boldsymbol{J}_{i}=0$ lead to:

$$
\phi \frac{\partial c}{\partial t}+\nabla \cdot(c \vartheta)=0
$$

This overall balance equation can replace the balance equation of one of the components in Eq. 1 such as the $n_{c}$-th component. The system of balance equations for $\left(n_{c}-1\right)$ components and the overall balance equation can then be expressed in the form:

$$
\begin{aligned}
& \phi \frac{\partial c}{\partial t}+\nabla \cdot(c \boldsymbol{\vartheta})=0, \\
& \phi \frac{\partial \varsigma_{i}}{\partial t}+\nabla \cdot\left(\varsigma_{i} \boldsymbol{\vartheta}\right)+\nabla . \boldsymbol{J}_{i}=0 \quad i=1, \ldots, n_{c}-1,
\end{aligned}
$$

This article is protected by copyright. All rights reserved. 
where, $\varsigma_{i}=c z_{i}$ for $i=1, \ldots, n_{c}-1$.

In this work, the reservoir boundaries are assumed impermeable. Therefore, the convection and diffusion fluxes across the outer boundaries are zero, i.e.,

$$
\begin{array}{ll}
\boldsymbol{\vartheta} . \boldsymbol{n}=0 & \text { on } \quad \Gamma, \\
\boldsymbol{J}_{i} . \boldsymbol{n}=0 & i=1, \ldots, n_{c}-1 \text { on } \quad \Gamma,
\end{array}
$$

where, $\Gamma$ denotes the outer boundary of $\Omega$ and $\boldsymbol{n}$ is the unit outward normal to $\Gamma$.

\section{NUMERICAL APPROXIMATION}

The DG and MFE methods are used to solve the coupled system of equations 2, 3, 6, and 7. In a previous work $[19,20,21,45]$, a detailed description of this method in approximating the pure convection flow equations $\left(\boldsymbol{J}_{i}=0\right)$ in homogeneous and fractured media was provided. In this work, we briefly review the numerical formulation and extend the method to approximate the diffusion fluxes. We focus on the calculation of diffusion and convection fluxes. The governing equations are solved by the implicit method for pressure and explicit method for composition (IMPEC).

The domain $\Omega$ is discretized into an unstructured triangular or rectangle mesh with no restrictions on the geometrical shape of the cells. We consider the following notations:

$$
K \text { : mesh cell, }
$$

$E$ : edge of cell $K$,

$N_{e}$ : number of edges for each cell $\left(N_{e}=3\right.$ or 4$)$,

$N_{K}$ : number of cells in a mesh,

$N_{E}$ : number of edges in a mesh.

The construction of the numerical process is organized as follows:

1. Discretization of Darcy's law by the MFE method,

2. Discretization of the flow equations by the DG method,

3. System construction.

\subsection{Discretization of Darcy's law}

In the MFE method, both the pressure and its gradient are approximated simultaneously; the velocity field is approximated in the so-called Raviart-Thomas space of the lowest order $\left(R T_{0}\right)$. With this method, the velocity over each cell can be expressed in terms of the fluxes across the edges. Additional details of the MFE method is presented in [19, 20, 21].

Using the Raviart-Thomas approximation space, the vectors $\boldsymbol{\vartheta}$ and $\boldsymbol{g}$ can be expressed as:

$$
\boldsymbol{\vartheta}=\sum_{E \in \partial K} q_{K, E} w_{K, E} \text { and } \boldsymbol{g}=\sum_{E \in \partial K} q_{K, E}^{g} w_{K, E},
$$

where $w_{K, E}$ is the $R T_{0}$ basis function, $q_{K, E}$ is the total volumetric convection flux across edge $E$, and $q_{K, E}^{g}$ is the flux from the gravitational force. The basis function $w_{K, E}$ has the following properties:

$$
\begin{gathered}
\nabla \cdot w_{E}=\frac{1}{|K|}, \\
w_{E} \cdot n_{E^{\prime}}=\left\{\begin{array}{c}
1 /|E| \text { if } E=E^{\prime} \\
0 \text { if } E \neq E^{\prime}
\end{array}\right.
\end{gathered}
$$

This article is protected by copyright. All rights reserved. 
Therefore, the velocity field over each cell is determined by the normal fluxes across the cell edges, see Eq. 8. Multiplying Eq. 2 by the test function $w_{K, E}$ and integrating by parts, the total flux $q_{K, E}$ through edge $E$ is given as a function of the cell average pressure $p_{K}$ and the edge average pressures $t p_{K, E}$ in cell $K$, i.e.,

$$
q_{K, E}=\alpha_{K, E} p_{K}-\sum_{E^{\prime} \in \partial K}\left(B_{K}^{-1}\right)_{E, E^{\prime}} t p_{K, E^{\prime}}-\beta_{K, E} E \in \partial K,
$$

$$
\text { where, }
$$

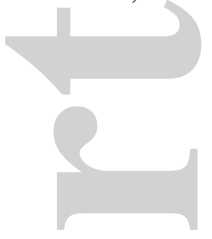

$$
\begin{array}{ll}
\left(B_{K}\right)_{E, E^{\prime}} & =\int_{K} w_{K, E} \mathbf{K}_{K}^{-1} w_{K, E^{\prime}} \\
\left(\tilde{B}_{K}\right)_{E, E^{\prime}} & =\int_{K} w_{K, E} w_{K, E^{\prime}} \\
\alpha_{K, E} & =\sum_{E^{\prime} \in \partial K}\left(B_{K}^{-1}\right)_{E, E^{\prime}} \\
\beta_{K, E} & =-\rho_{K} \sum_{E^{\prime} \in \partial K}\left(B_{K}^{-1} \tilde{B}_{K}\right)_{E, E^{\prime}} q_{K, E^{\prime}}^{g}
\end{array}
$$

Note that the coefficients $\left(B_{K}\right)_{E, E^{\prime}},\left(\tilde{B}_{K}\right)_{E, E^{\prime}}$, and $\alpha_{K, E}$ depend on the geometrical shape of the elements and the local mobility coefficient. By imposing the flux continuity across the cell interfaces, $\left(q_{K, E}=q_{K^{\prime}, E} ; \quad E=K \cap K^{\prime}\right)$, one can eliminate the flux unknowns $q_{K, E}$ from Eq. 11. As a result, the following linear system with primary unknowns; the cell average pressures in $P$ and the edge average pressures in $T_{P}$ becomes:

$$
R^{T} P-M T_{P}-I=0,
$$

\section{where,$$
\text { where, }
$$

$$
\begin{aligned}
& R=\left[R_{K, E}\right]_{N_{K}, N_{E}} \quad ; \quad R_{K, E}=\alpha_{K, E} \quad E \in \partial K, \\
& M=\left[M_{E, E^{\prime}}\right]_{N_{E}, N_{E}} \quad ; \quad M_{E, E^{\prime}}=\sum_{E, E^{\prime} \supset \partial K}\left(B_{K}^{-1}\right)_{E, E^{\prime}}, \\
& I=\left[I_{E}\right]_{N_{E}} \quad ; \quad I_{E}=\left\{\begin{array}{l}
\beta_{K, E} \quad E \in \Gamma \\
\beta_{K, E}+\beta_{K^{\prime}, E}
\end{array} \quad E=K \cap K^{\prime}\right.
\end{aligned}
$$

\subsection{Discretization of the flow equations}

The DG method is used to discretize the flow equations, Eq. 6. For simplicity, we apply the DG method to the flow equation of component $i\left(i<n_{c}\right)$. Over each cell $\mathrm{K}$, the unknown $\varsigma$ is approximated in a piecewise discontinuous, linear (on triangles) or bilinear (on quadrilaterals) finite element space, so that,

$$
\varsigma_{K, i}=\sum_{j=1}^{N_{e}} \varsigma_{K, i}^{j} \varphi_{K, j},
$$

where $\varphi_{K, j}$ is the finite element shape functions. Multiplying the flow Eq. 6 by the test functions $\varphi_{K, l}$ and integrating by parts produces volume and surface integrals that have to be evaluated over each cell, i.e.,

$$
\begin{aligned}
\int_{K} \phi \frac{\partial \varsigma_{K, i}}{\partial t} \varphi_{K, l} & -\int_{K} \varsigma_{K, i} \boldsymbol{\vartheta} \cdot \nabla \varphi_{K, l}+\int_{\partial K} \varsigma_{K, i} \varphi_{K, l} \boldsymbol{\vartheta} . \boldsymbol{n} \\
& -\int_{K} \boldsymbol{J}_{i} \cdot \nabla \varphi_{K, l}+\int_{\partial K} \varphi_{K, l} \boldsymbol{J}_{i} \cdot \boldsymbol{n}=0 \quad l=1, \ldots, N_{e} .
\end{aligned}
$$

The volume integrals in Eq. 14 can be readily calculated. However, due to the discontinuity of the functions at finite element boundaries, the surface integrals describing the convection and diffusion fluxes have to be properly defined. The convection and diffusion fluxes are modeled differently, as described in the following sections. 
3.2.1. Convection fluxes An accurate convection flux is essential for properly simulating the flow equations. For this reason, we choose to approximate the flow velocity by the MFE method. Using Eq. 10, the convection flux in Eq. 14 simplifies to:

$$
\begin{aligned}
\int_{\partial K} \varsigma_{K, i} \varphi_{K, l} \boldsymbol{\vartheta} \cdot \boldsymbol{n} & =\int_{\partial K} \varsigma_{K, i} \varphi_{K, l}\left(\sum_{E} q_{K, E} w_{K, E}\right) \cdot \boldsymbol{n} \\
& =\sum_{E} q_{K, E} \int_{E} \varsigma_{K, i} \varphi_{K, j} w_{K, E} \cdot \boldsymbol{n}_{E}=\sum_{E} \frac{q_{K, E}}{|E|} \int_{E} \varsigma_{K, i} \varphi_{K, j} .
\end{aligned}
$$

Since the concentration variable $\left.\varsigma_{i, K}\right|_{E}$ at the cell boundary $E$ is allowed to be discontinuous, this term is treated by an upwind scheme, i.e.,

$$
\left.\varsigma_{i, K}\right|_{E}=\left\{\begin{array}{l}
\varsigma_{i, K}^{i n} \text { if } q_{K, E}>0 \\
\varsigma_{i, K}^{\text {out }} \text { otherwise }
\end{array}\right.
$$

The two-point unwinding technique is sufficient to keep the DG method stable when using piecewise constant approximations. However, for higher order approximations, an efficient slope limiter is need $[19,46]$.

The volumetric flux is computed from Eq. 11 as a function of the cell average pressure and the average edge pressures. For structured grids, one can prove that the flux in the MFE with some approximations of the integrals is equivalent to the flux in the FD method [22]. However, for unstructured grids, the FD method looses accuracy. For gas injection problems, we showed in [19] that using the two-point FD technique to approximate fluxes as an alternative to the MFE method can be useful in grids of good quality (such as equilateral triangles). This study shows that, to model natural convection, the FD approximation leads to inaccuracies in the velocity field even when using grids with good quality. The main reason is that, with natural convection the velocity field is often very small (in the order of $10^{-10} \mathrm{~m} / \mathrm{s}$ ). Therefore, to get meaningful simulations, the numerical errors generated by method have to be smaller in magnitude than the velocity values, which was found to be a challenge for the FD method. A numerical example (Example 1) is introduced to clarify this statement.

3.2.2. Diffusion fluxes Using the expression for $\boldsymbol{J}_{i}$, the total diffusion flux in Eq. 4 is expanded to:

$$
\int_{K} \varphi_{K, l} \boldsymbol{J}_{i} \cdot \boldsymbol{n}=-\sum_{j=1}^{n_{c}-1} \int_{\partial K} \varphi_{K, l} \tilde{D}_{i j}^{M} \nabla z_{j} . \boldsymbol{n}-\int_{\partial K} \varphi_{K, l} \tilde{D}_{i}^{p} \nabla p . \boldsymbol{n}-\int_{\partial K} \varphi_{K, l} \tilde{D}_{i}^{T} \nabla T \cdot \boldsymbol{n},
$$

where, $\tilde{D}_{i j}^{M}=c D_{i j}^{M}, \tilde{D}_{i}^{p}=c D_{i}^{p}$, and $\tilde{D}_{i}^{T}=c D_{i}^{T}$. The thermal, pressure and Fickian fluxes are approximated differently as described below.

\section{Thermal diffusion flux}

The thermal diffusion is expressed using the integral:

$$
f_{i}^{T}=-\int_{\partial K} \varphi_{K, l} \tilde{D}_{i}^{T} \nabla T \cdot \boldsymbol{n} .
$$

We assume that the temperature $T$ is known in the domain. Usually, $T$ is considered to be a linear function of the spatial coordinates. The coefficient $\tilde{D}_{i}^{T}$ at the cell interface $E$ is determined according to the upstream direction of the driving force, i.e.,

$$
\left.\tilde{D}_{i}^{T}\right|_{E}=\left\{\begin{array}{l}
\tilde{D}_{i}^{T, \text { in }} \text { if } \nabla T \cdot \boldsymbol{n}_{E}>0 \\
\tilde{D}_{i}^{T, \text { out }} \text { otherwise }
\end{array}\right.
$$

This article is protected by copyright. All rights reserved. 
Pressure diffusion flux

The pressure diffusion flux is expressed by:

$$
f_{i}^{p}=-\int_{\partial K} \varphi_{K, l} \tilde{D}_{i}^{p} \nabla p . \boldsymbol{n} .
$$

The MFE approximation allows expressing the pressure gradient vector by flux $q_{K, E}^{p}$ across the edge, so that:

$$
-\nabla p=\sum_{E \subset \partial K} q_{K, E}^{p} w_{K, E} .
$$

Similarly to Eq. $11, q_{K, E}^{p}$ is written as a function of the cell average pressure and the pressure traces:

$$
q_{K, E}^{p}=\tilde{\alpha}_{K, E} p_{K}-\sum_{E^{\prime} \in \partial K}\left(\tilde{B}_{K}^{-1}\right)_{E, E^{\prime}} t p_{K, E^{\prime}} \quad E \in \partial K,
$$

where, $\left(\tilde{B}_{K}\right)_{E, E^{\prime}}=\int_{K} w_{K, E} w_{K, E^{\prime}}$ and $\tilde{\alpha}_{K, E}=\sum_{E^{\prime} \in \partial K}\left(\tilde{B}_{K}^{-1}\right)_{E, E^{\prime}}$. Using Eqs. 20 and 10, we get:

$$
f_{i}^{p}=\sum_{E} \frac{q_{K, E}^{p}}{|E|} \int_{E} \tilde{D}_{i}^{p} \varphi_{K, j} .
$$

This expression is similar to the convection flux given in Eq. (15). The coefficient $\tilde{D}_{i}^{p}$ is evaluated as:

$$
\left.\tilde{D}_{i}^{p}\right|_{E}=\left\{\begin{array}{l}
\tilde{D}_{i}^{p, \text { in }} \text { if } q_{K, E}^{p}>0 \\
\tilde{D}_{i}^{p, \text { out }} \text { otherwise }
\end{array}\right.
$$

We note that the pressure unknown in Eq. 22 is modeled implicitly.

\section{Molecular diffusion flux}

We express the molecular diffusion flux of component $i$ as follows:

$$
f_{i}^{M}=-\int_{\partial K} \varphi_{K, l} \tilde{D}_{i i}^{M} \nabla z_{i} . \boldsymbol{n} .
$$

Other fluxes for cross-diffusion are treated in a similar way. We present two distinct approaches to approximate Eq. 24 by both the MFE and LDG methods. Each approach has advantages and drawbacks as explained below.

\section{Diffusion flux by the MFE method}

We denote by $z_{K}=\frac{1}{|K|} \int_{K} z_{i}, \quad t z_{K, E}=\frac{1}{|E|} \int_{E} z_{i}$ the cell and edge averages of $z_{i}$, respectively. Note that the subscript $i$ is dropped to simplify the notation. We also introduce an auxiliary variable $\vartheta^{z}$ :

$$
\vartheta^{z}=-\tilde{D}_{i i}^{M} \nabla z_{i} .
$$

Using the Raviart-Thomas basis function, $\vartheta^{z}$ can be expressed in terms of the flux unknowns:

$$
\vartheta^{z}=\sum_{E} q_{K, E}^{z} w_{K, E}
$$

This article is protected by copyright. All rights reserved. 
Multiplying Eq. 25 by function $w_{K, E}$ and integrating by parts yields:

$$
q_{K, E}^{z}=\alpha_{K, E}^{z} z_{K}-\sum_{E^{\prime} \in \partial K}\left(B_{K}^{-1}\right)_{E, E^{\prime}}^{z} t z_{K, E^{\prime}} \quad E \in \partial K,
$$

where $\left(B_{K}\right)_{E, E^{\prime}}^{z}=\int_{K} \tilde{D}_{i i}^{M} w_{K, E} w_{K, E^{\prime}}$ and $\tilde{\alpha}_{K, E}^{z}=\sum_{E^{\prime} \in \partial K}\left(B_{K}^{-1}\right)_{E, E^{\prime}}^{z}$

Following the same technique used to discretize Darcy's law, we impose the continuity of the fluxes $q_{K, E}^{z}$ to construct the following linear system:

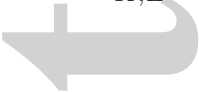

$$
\hat{M} T_{Z}=\hat{R}^{T} Z,
$$

where,

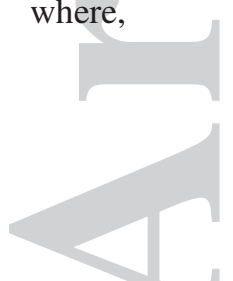

$$
\begin{aligned}
& \hat{R}=\left[\hat{R}_{K, E}\right]_{N_{K}, N_{E}}, \quad \hat{R}_{K, E}=\alpha_{K, E}^{z} \quad E \in \partial K \\
& \hat{M}=\left[\hat{M}_{E, E^{\prime}}\right]_{N_{E}, N_{E}}, \quad \hat{M}_{E, E^{\prime}}=\sum_{E, E^{\prime} \supset \partial K}\left(B_{K}^{-1}\right)_{E, E^{\prime}}^{z} \\
& Z=\left[z_{K}\right]_{N_{K}}, \text { and } T_{Z}=\left[t z_{E}\right]_{N_{E}} .
\end{aligned}
$$

The procedure is then complete to compute the fluxes. For a given compositional distribution $Z, T_{Z}$ is calculated by solving the linear system in Eq. 28 . The flux $q_{K, E}^{z}$ can then be computed locally from Eq. 27. Although the matrix $\hat{M}$ is symmetric and a positive definite, solving Eq. 28 for each time step and for each component can potentially be expensive. However, by using the upstream values of the coefficients at the cell boundaries, $\hat{M}$ becomes dependent on the finite element geometries only. Therefore, one way to accelerate the calculation is to store the Cholesky decomposition of $\hat{M}$ (once, at the start of the simulation); the calculation of the fluxes for each component is then reduced to the cost of solving two triangular linear systems. In the following section, we present an alternative approach to evaluate Eq. 24 by using the LDG method.

\section{Diffusion fluxes by the LDG method}

The LDG method possesses features that are similar to the MFE method. One of them is that the primary unknown and its gradient are approximated simultaneously. The LDG method conserves mass locally and is flexible for high-order approximations. The main difference between the LDG and MFE methods is that, in the LDG method, the fluxes can be calculated locally, whereas different fluxes approximated by the MFE are computed through a global linear system. For each cell $K$, we introduce the auxiliary variable $v_{K}$ so that:

$$
v_{K}=-\tilde{D}_{i i}^{M} \nabla z_{i, K} .
$$

Letting $v_{K}=\left(q_{x, K}, q_{y, K}\right)^{T}$ and substituting into Eq. 24, we obtain:

$$
\begin{aligned}
f_{i}^{M} & =\int_{\partial K} \varphi_{K, l} v_{K} \cdot \boldsymbol{n}_{K} \\
& =\int_{\partial K} \varphi_{K, l}\left(q_{x, K} n_{x, K}+q_{y, K} n_{y, K}\right)
\end{aligned}
$$

Where, $\boldsymbol{n}_{K}=\left(n_{x, K}, n_{y, K}\right)$ is the outward unit vector to the boundary $E$. Due to the discontinuity of $v_{K}$ at the interface, Eq. 30 is replaced by:

$$
\begin{aligned}
f_{i}^{M} & =\int_{\partial K} \varphi_{K, l} \hat{v} \cdot n_{K} \\
& =\int_{\partial K} \varphi_{K, l}\left(\hat{q}_{x} n_{x, K}+\hat{q}_{y} n_{y, K}\right) .
\end{aligned}
$$

This article is protected by copyright. All rights reserved. 
Multiplying Eq. 30 by a test function $\varphi$ and integrating by parts, we obtain:

$$
\begin{gathered}
\int_{K} \frac{1}{\tilde{D}_{i i}^{M}} q_{x, K} \varphi=\int_{K} z_{i, K} \frac{\partial \varphi}{\partial x}-\int_{\partial K} \varphi \hat{z}_{i} n_{x, K}, \\
\int_{K} \frac{1}{\tilde{D}_{i i}^{M}} q_{y, K} \varphi=\int_{K} z_{i, K} \frac{\partial \varphi}{\partial y}-\int_{\partial K} \varphi \hat{z}_{i} n_{y, K} .
\end{gathered}
$$

The terms $\hat{v}$, and $\hat{z}_{i}$ (with "hats") are the numerical fluxes at the boundary that link the two elements. A crucial aspect of the LDG method for stability is the proper choice of these numerical fluxes. Different choices are possible to define the numerical fluxes; here we adopt the formulation given in [46]. At each edge $E$ separating two cells $K$ and $K^{\prime}\left(E=K \cap K^{\prime}\right)$, the unknowns $z_{i}$ and $v$ calculated within $K$ and $K^{\prime}$ are discontinuous. The numerical fluxes are defined in terms of the jumps and the averages at the cell boundaries. The jump and average are defined separately for scalars and vectors. For a vector, the jump and average at $E$ are, respectively, defined as follows:

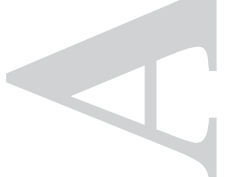

$$
\begin{aligned}
& \left\{z_{i}\right\}=\frac{1}{2}\left(z_{i, K}+z_{i, K^{\prime}}\right) \\
& {\left[z_{i, K}\right]=z_{i, K} \boldsymbol{n}_{K}+z_{i, K^{\prime}} \boldsymbol{n}_{K^{\prime}}}
\end{aligned}
$$

For a scalar, the jump and average are:

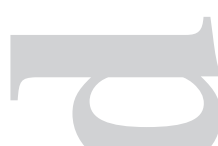

$$
\begin{aligned}
& \{v\}=\frac{1}{2}\left(v_{K}+v_{K^{\prime}}\right) \\
& {[v]=v_{K} \cdot \boldsymbol{n}_{K}+v_{K} \cdot \boldsymbol{n}_{K^{\prime}}}
\end{aligned}
$$

Note that the jump of a scalar is a vector and that the jump of a vector is a scalar. The numerical fluxes appearing in Eqs. 31, 32 are then:

$$
\hat{z}_{i}=\left\{z_{i}\right\}+\gamma \cdot\left[z_{i}\right], \quad \hat{v}=\{v\}-\beta \cdot[v] .
$$

Here, we set $\gamma=(1,1)$ and $\beta=1$ to achieve an optimal convergence [47]. These terms control the introduced numerical dispersion necessary to keep the method numerically stable. By neglecting these terms and using structured grids, the method reduces to the conventional cell-centered FD method[37]. Using Eqs. 32 and 35, the numerical fluxes can be computed by solving a local linear system. However, due to the discontinuity of velocity at the inter-elements, the LDG method loses accuracy as compared to the MFE method. A numerical test in Example 2 is devoted to compare the MFE and LDG methods.

\subsection{System construction}

The Newton-Raphson method is used to linearize the coupled systems from Eqs. 3, 12, and 15. An IMPEC scheme is implemented in which the pressure unknowns are modeled implicitly and the composition unknowns are modeled explicitly in time. The diffusion fluxes, except for the pressure diffusion, are explicitly in time and therefore they are treated as a sink/source terms. A full description of the algorithm is provided in references [19, 20, 21, 45].

\section{NUMERICAL RESULTS}

In this section, we present the results of calculations from the algorithm described above for various examples covering homogeneous, heterogeneous, and fractured media. Two-, threeand ten-component fluid mixtures are used in the examples. One of the examples is a tilted reservoir.

Example 1: In this example, we investigate the accuracies of the velocity fields approximated by the MFE and the conventional two-point FV method. We consider a rectangular cross-sectional domain 
saturated with a hydrocarbon mixture of $C_{1} / n C_{4}$ (see Table I). The permeability in the domain is $1 \mathrm{md}$, except in one zone where its value is $10^{-3} \mathrm{md}$ (see Fig. 1). The domain is discretized into an unstructured triangular mesh. Two different cases are considered. In the first case, no fluid injection was considered and the fluid flow is solely from natural convection. Fig. 1a shows the velocity field from the two-point FV approximation. Although, the mesh quality is good as all triangular cells are almost equilateral, inaccuracy in the velocity field is clearly observed. In Fig. 1b, the velocity field approximated by the MFE method has no apparent inaccuracy. The poor accuracy of the FV method is related to the magnitude of the velocity field which is apparently within the order of magnitude of the numerical error introduced by the method (approximately $5 \times 10^{-10} \mathrm{~m} / \mathrm{s}$ ). To examine this hypothesis, we introduce a second case where the velocity is increased in the domain by injecting $C_{1}$ in the left lower corner and producing $C_{1} / n C_{4}$ from the diagonally opposite corner. The injection rate is $10^{-2} \mathrm{~m}^{3} / \mathrm{day}$, and the velocity is in the order $10^{-7} \mathrm{~m} / \mathrm{s}$. In this case, the FV and MFE methods provide nearly the same results. Fig. 2 shows the velocity fields calculated by both methods. We conclude that a method with a higher order of approximation such as the MFE method is required to accurately approximate the velocity field in natural convection.

Example 2: In this example, we investigate the accuracy of MFE and LDG methods in calculating the Fickian diffusion flux. The main advantage of the LDG method is in its flexibility to compute the fluxes locally with a high-order approximation. For computational efficiency, we use a first-order approximation. In the DG method, local linear systems are solved to compute the fluxes. In the MFE method, however, a global linear system is solved to compute the fluxes. The LDG method is more efficient, but it is generally less accurate than the MFE method. The superiority of the MFE method has been reported in [47].

We consider a vertical domain of dimensions $\left(L_{z}=100 m, L_{x}=\Delta x m\right)$ saturated with a binary mixture $C_{1} / n C_{4}$ (Table I). The domain is discretized into one column of right angled-triangles (see Fig. 3a). We fix the number of cells in the mesh (i.e., fixed $\Delta z$ ) and vary the column base, $\Delta x$. The accuracies of the LDG and MFE methods are investigated when the ratio $\kappa=\Delta x / \Delta z$ is varied. When $\kappa$ increases, the triangles tend to flatten. In Fig. $3 \mathrm{~b}, C_{1}$ composition versus height calculated from the LDG and MFE methods are presented for different values of $\kappa$. The change in $C_{1}$ composition profiles as a function of $\kappa$ in the LDG solution is unphysical. This variation is a result of mesh dependency. On the other hand, the MFE method shows almost no mesh dependency (Fig. 3b). The mesh dependency can be further examined by showing $C_{1}$ composition at a fixed point in the domain for different values of $\kappa$. Figure 4 demonstrates that there is very little change in the MFE solution compared to the LDG solution. The example illustrates the superiority of the MFE method in approximating the diffusion flux in distorted grids. For good quality grids, the LDG method is preferred because of its superior efficiency. In this example, the LDG method was 40 to $50 \%$ faster than the MFE method.

Example 3: In this example, we investigate the permeability effect on the compositional variation of a binary mixture of methane and normal butane in a vertical cross-section $\left(L_{z}=150 \mathrm{~m}, L_{x}=3000 \mathrm{~m}\right)$. The input data are provided in Table I. The diffusion coefficients, $D_{i j}^{M}, D_{i}^{p}$, and $D_{i}^{T}$ (see Eq. (4)), are calculated as a function of pressure, temperature and composition. The methane composition contours at steady state, for different values of permeability $(k=0,0.5,2,30,1000 \mathrm{md})$, are presented in Fig. 5. The results are in good agreement with those presented in [15]. The increase of permeability $k$ has only a minor effect on the performance of our algorithm performance. Methane tends to segregate more in the hotter region of the domain mainly due to the thermal diffusion in Fig. 5a. For high permeabilities $(k>30 m d)$, the natural convection becomes more dominant, almost leading to a homogeneous distribution of the fluid (see Fig. $5 \mathrm{~d}$ and Fig. 5e). Fig. 5f shows the velocity distribution in the domain for $k=1000 \mathrm{md}$, for calculations performed on a structured mesh with 671 elements. The CPU time is about 250s with permeability, $k=1000 \mathrm{md}$. All calculations were performed on an Intel® Xeon 64bit PC. Although the increase of permeability reduces the time step size because of the CFL condition, the CPU time required for the other cases $(k=0.5,2,30 \mathrm{md})$ is of the same order. With lower permeability, more iterations on

This article is protected by copyright. All rights reserved. 
the transient equations were needed to reach the steady state.

Example 4: In this example, we compute the compositional variation in a vertical crosssection for two different mixtures. Our results are compared with the work of Ghorayeb and Firoozabadi [17]. In Example $4 \mathrm{a}$, we use a ternary mixture of $C_{1} / C_{2} / n C_{4}$, and in Example $4 \mathrm{~b}$, a 10-component mixture. Tables II and III provide respective relevant data. In all our simulations, the diffusion coefficients, as well as the density, are allowed to vary in space and in time, unlike in the numerical model introduced in [17]. In Example 4a, the composition of the ternary mixture at the center of the reservoir is: $C_{1} 25 \%, C_{2} 25 \%$, and $C_{3} 50 \%$ (moles). In Fig. 6, the composition contour plots for $C_{1}$ are depicted for different permeabilities $(k=0,1,10,100 \mathrm{md})$. We observe that $C_{1}$ is more segregated in the hot area on the right side of the domain, similarly to what we observe for a binary mixture. There is almost no variation in composition with an increase in permeability. In all runs, calculations are performed on a uniform grid of 671 elements. In this run and all the other runs, there is practically no change in the results from finer gridding. The CPU time was about 400s. In this run and all the other runs, there was practically no change in the results form finer gridding. Example $4 \mathrm{~b}$ presents the results obtained with large scale field dimensions; relevant data are listed in Table III. Fig. 7 shows composition profiles of $C_{1}, C_{5}-C_{6}$, and $C_{30+}$ for permeabilities $k=0$ (convection free) and $k=10 \mathrm{md}$. The results are in good agreement with those in [15]. Methane, which has the maximum variation, segregates towards the top of the domain. However, the other components, except $C_{30+}$, have no significant compositional variation. Note that natural convection reduces segregation. The domain is discretized into a rectangular mesh consisting of 3321 elements. The CPU time was about 3200s.

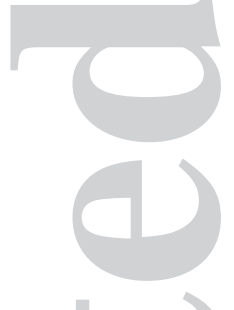

Example 5: Ghorayeb and Firoozabadi [18] investigated the effect of fractures on the compositional variation of binary systems. They studied natural convection and diffusion in

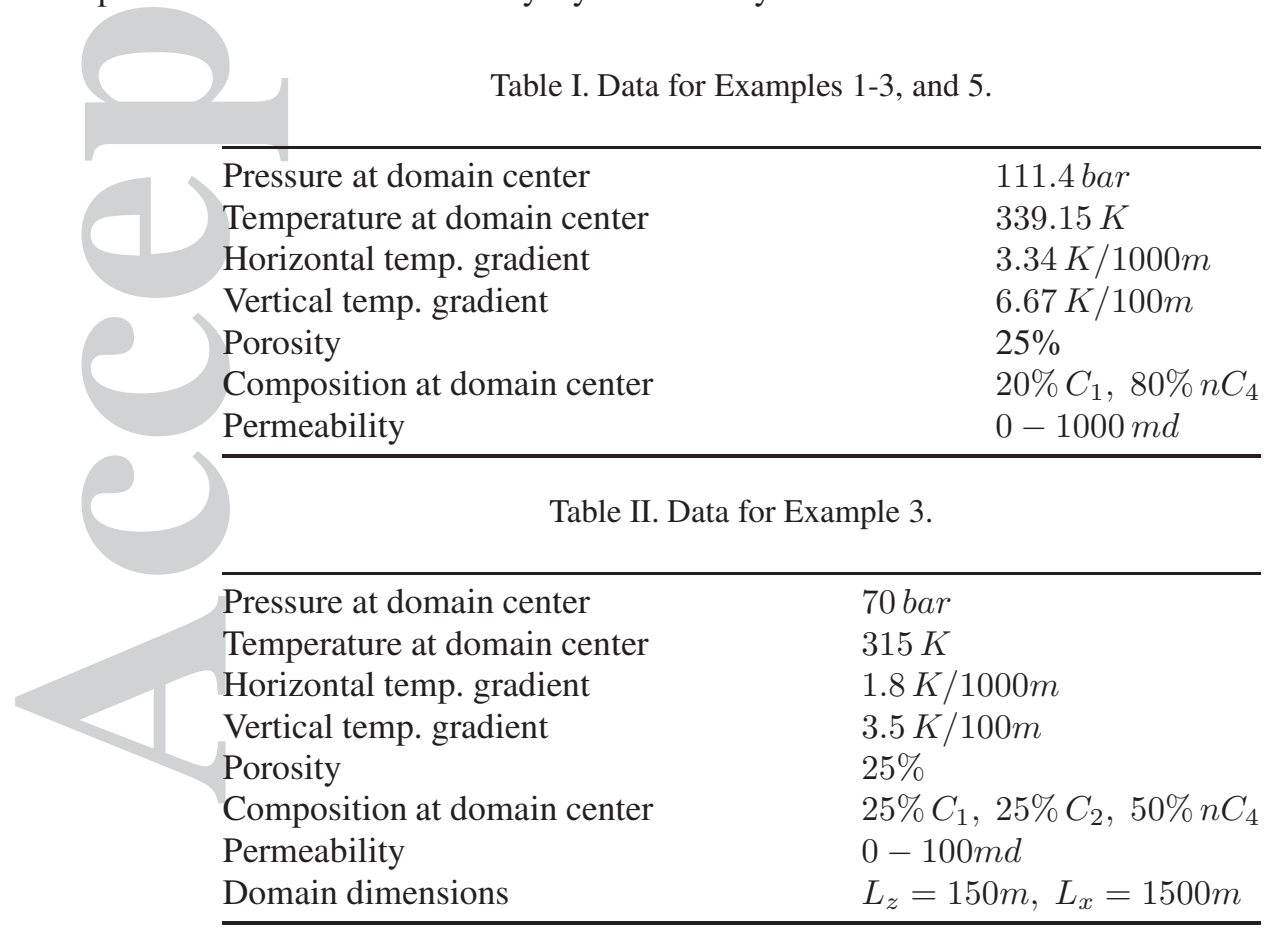

This article is protected by copyright. All rights reserved. 


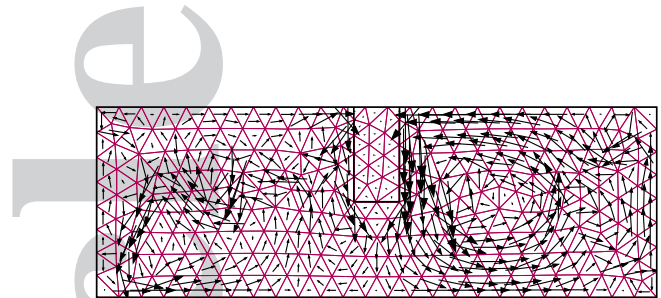

a) Two-point FV

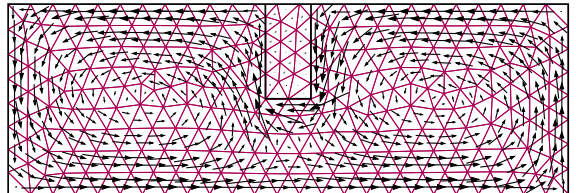

b) MFE

Figure 1. Velocity field by the FV and MFE methods; natural convection; Max Vel. $\approx 5 \times 10^{-10} \mathrm{~m} / \mathrm{s}$; Example 1, Case 1.

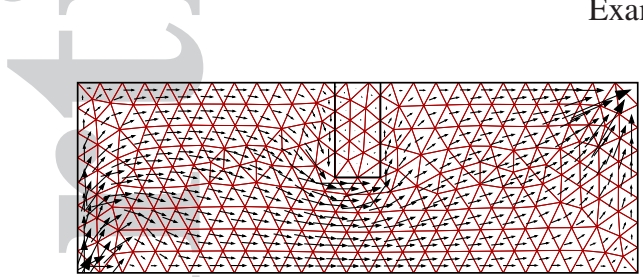

a) Two-point FV

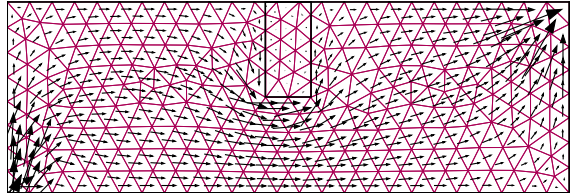

b) $\mathrm{MFE}$

Figure 2. Velocity field by the FV and MFE methods; gas injection at the lower left corner, fluid production through the opposite corner; Max Vel. $\approx 10^{-7} \mathrm{~m} / \mathrm{s}$; injection rate $=10^{-2} \mathrm{~m}^{3} /$ day; Example 1 , Case 2.

fractured media by using the single-porosity model in FD in which the fractured medium is treated as a heterogeneous medium with distinct matrix and fracture permeabilities. The authors reported

\begin{tabular}{|c|c|}
\hline \multicolumn{2}{|c|}{ Table III. Data for Example 4.} \\
\hline Pressure at domain center & 466 bar \\
\hline Temperature at domain center & $422 K$ \\
\hline Horizontal temp. gradient & $1.5 \mathrm{~K} / 1000 \mathrm{~m}$ \\
\hline Vertical temp. gradient & $2.75 \mathrm{~K} / 100 \mathrm{~m}$ \\
\hline Porosity & $25 \%$ \\
\hline Composition at domain center & $3.229 \% \mathrm{CO}_{2}$ \\
\hline & $62.53 \% C_{1}$ \\
\hline I & $9.644 \% C_{2}$ \\
\hline & $9.080 \% C_{3}-n C_{4}$ \\
\hline & $3.436 \% C_{5}-C_{6}$ \\
\hline & $5.979 \% C_{7}-C_{10}$ \\
\hline & $2.450 \% C_{11}-C_{14}$ \\
\hline & $2.085 \% C_{15}-C_{20}$ \\
\hline & $1.288 \% C_{21}-C_{29}$ \\
\hline & $0.556 \% C_{30+}$ \\
\hline Permeability & $0-10 m d$ \\
\hline Domain dimensions ix $\mathrm{x}-\mathrm{z}$ plane & $L_{z}=1500 \mathrm{~m}, L_{x}=10000 \mathrm{~m}$ \\
\hline
\end{tabular}

Table IV. Data for Example 6.

\begin{tabular}{ll}
\hline Pressure at domain center & \\
Temperature at domain center & $890 \mathrm{bar}$ \\
Horizontal temp. gradient & $360.1 \mathrm{~K}$ \\
Vertical temp. gradient & $1.8 \mathrm{~K} / 1000 \mathrm{~m}$ \\
Porosity & $3.5 \mathrm{~K} / 100 \mathrm{~m}$ \\
Composition at domain center & $25 \%$ \\
Permeability & $20 \% \mathrm{C}_{1}, 80 \% \mathrm{nC}_{4}$ \\
\hline
\end{tabular}

This article is protected by copyright. All rights reserved. 

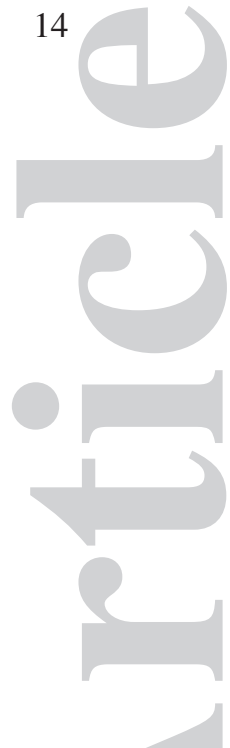

a) Mesh

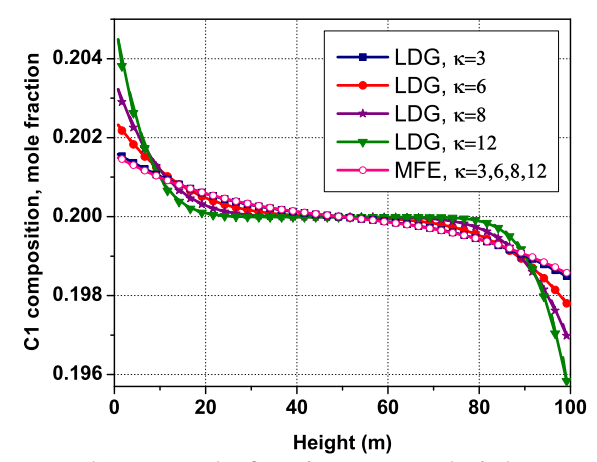

b) $C_{1}$ mole fraction versus height

Figure 3. Mesh consisting of 1 column of right-angled triangles and distribution of $C_{1}$ composition computed by the LDG and MFE methods as a function of the ratio $\kappa$; Example 2.
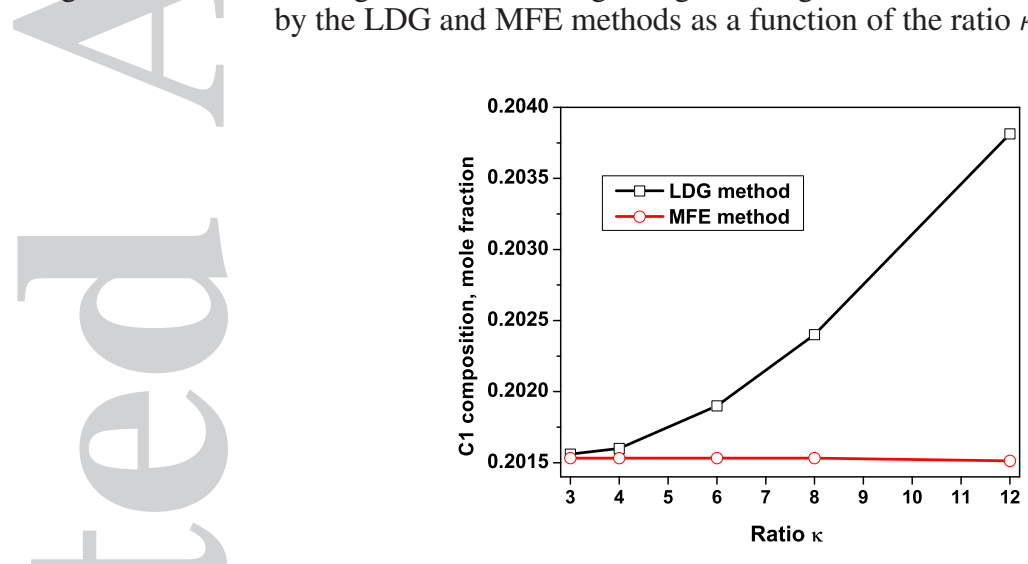

Figure 4. Variation of $C_{1}$ composition as a function of $\kappa$ at a fixed point (at height $=0$ ) in the domain by using the LDG and MFE methods; Example 2.

that the discretization of the governing equations by the FD model led to ill-conditioned large systems because of: 1) a sharp variation in permeability between the matrix and fractures, 2) a small fracture aperture compared to the matrix size. Therefore, the single-porosity approach has limitations from a numerical perspective. The discrete fracture model appears to be an attractive, simpler alternative to the single porosity model where fractures are represented by $(n-1)$-dimensional elements in an n-dimensional domain. We compute the compositional variation of a binary mixture $C_{1} / n C_{4}$ in a rectangular domain of $15 \mathrm{~m} \times 30 \mathrm{~m}$ where the matrix permeability is $k_{m}=1 \mathrm{md}$ and the fracture aperture is $f_{a}=1 \mathrm{~mm}$ (see Table IV). Several configurations of fractures are used to study the effect of the fracture connectivity and that of the fracture permeability, which varies from $k_{f}=1 m d$ (no fractures) to $k_{f}=10^{5} \mathrm{md}$ (Fig. 8). In configuration 1 (Fig. 8a), one fracture is considered around the domain boundaries. In configurations 2 and 3 , (Figs. $8 \mathrm{~b}$ and 8c) the fractures are disconnected, and configuration 4 (Fig. 8d) represents a sugar cube geometry consisting of 50 matrix blocks surrounded by fractures. The methane contour lines with low fracture permeabilities are shown as references in configuration 1 (Fig. 9). Fig. 10 shows the methane distribution and velocity field, at steady state, for all configurations with fracture permeabilities of $10^{4}$ and $10^{5} \mathrm{md}$. In Fig. 10b, the velocity field consists mainly of one loop. The results show that the increase in fracture permeability makes the composition uniform, as expected. Disconnected fractures in configurations 2 and 3 (Figs. 10d and 10f) have less effect on compositional variation. In configuration 4 (Fig. 10h), composition distributions show that, unlike in configuration 1, the mole fraction contours take an 'S' shape when the fracture permeability 


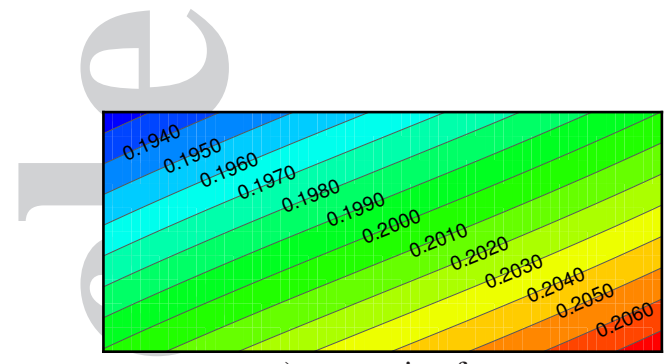

a) convection free

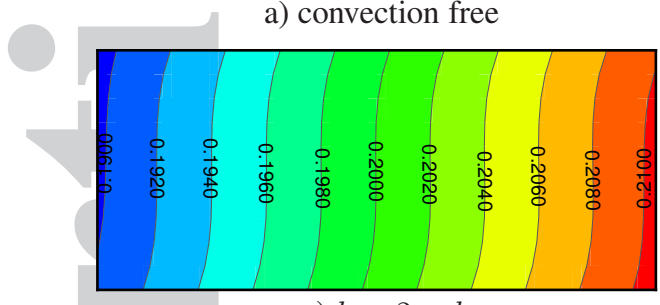

c) $k=2 m d$

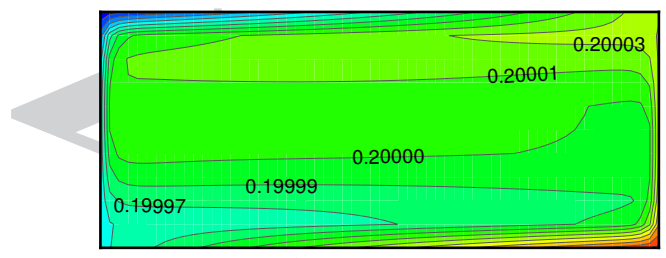

e) $k=1000 m d$

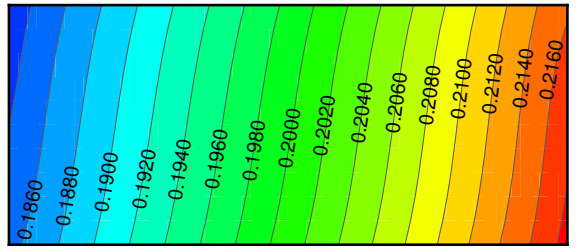

b) $k=0.5 \mathrm{md}$

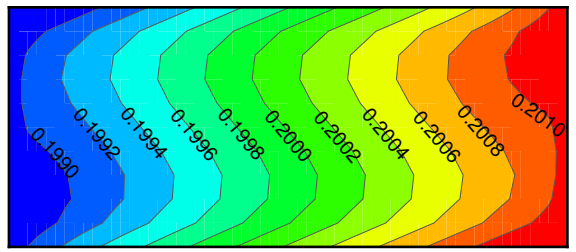

d) $k=30 \mathrm{md}$

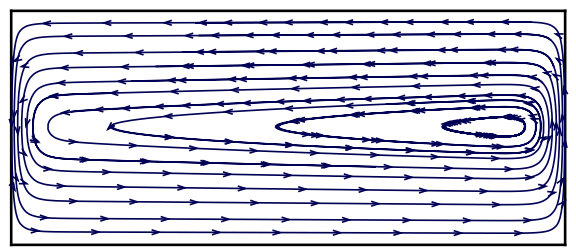

f) velocity field for $k=1000 \mathrm{md}$

Figure 5. Contours of methane composition (mole fraction) for different permeabilities and velocity field corresponding to $k=1000 \mathrm{md}$, binary mixture at steady state ; $(L x=3000 \mathrm{~m} ; L z=150 \mathrm{~m})$; Example 3 .

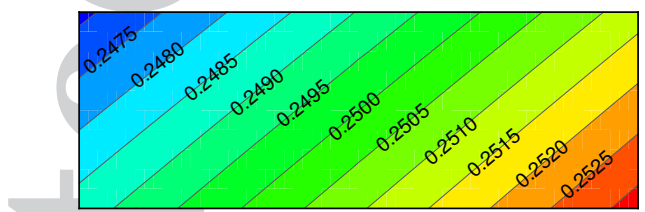

a) convection free

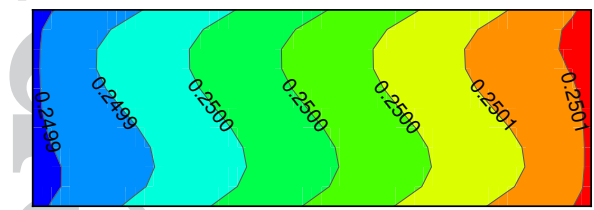

c) $k=10 \mathrm{md}$

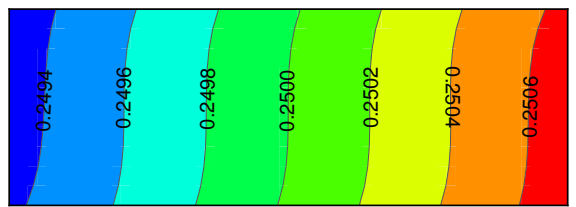

b) $k=1 \mathrm{md}$

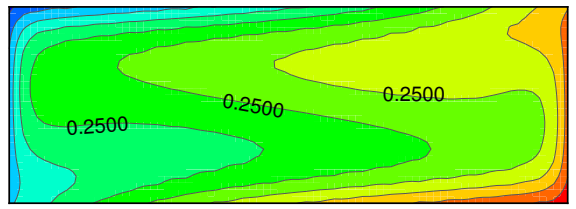

d) $k=100 \mathrm{md}$

Figure 6. Composition contour plot for $C_{1}$, with different permeability cases, ternary mixture $\left(25 \% C_{1}, 25 \% C_{2}, 50 \% n C_{4}\right.$, at the center $) ;(L x=1500 m ; L z=150 m)$; Example $4 \mathrm{a}$.

increases. Our results are in good agreement with those reported in [17]. One advantage of our model is that the decrease in fracture thickness does not impact the computational efficiency. However, in the single-porosity model, as the contrast between the matrix and fractures increases, more refined gridding is needed. We note that the CPU time required for our calculations in configuration 4 is about $150 \mathrm{~s}$ in a uniform grid of 450 elements. The CPU times for the other cases are less than 3 minutes. The performance of our model is 2-3 orders of magnitude faster than the FD-based single-porosity model. The speed-up is essentially due to a smaller number of grid cells needed in our model.

To show the robustness of our model, we increase the domain dimensions $(150 \mathrm{~m} \times 6000 \mathrm{~m})$. The fracture aperture and permeability are, respectively, $f_{a}=0.1 \mathrm{~mm}$ and $k_{f}=10^{4} \mathrm{md}$. We consider three configurations: 1) homogeneous, 2) fractured with a sugar cube geometry, 3) partially fractured domain with two subdomains, one unfractured side and the other fractured with sugar

This article is protected by copyright. All rights reserved. 


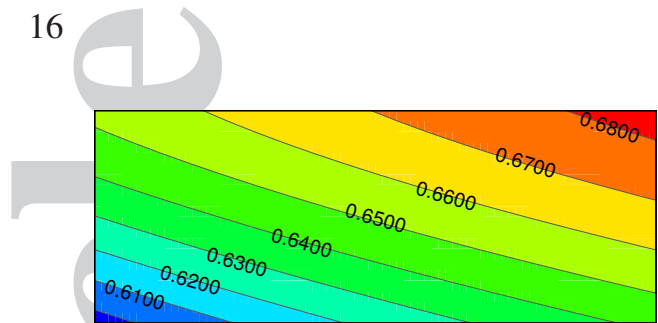

a) $C_{1}$, convection free

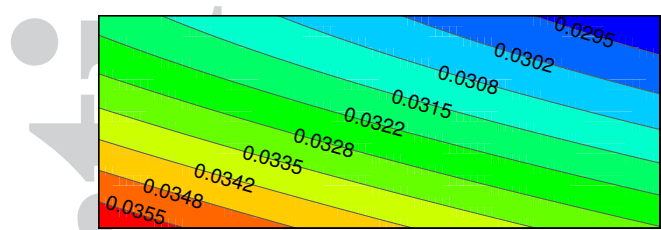

c) $C_{5}-C_{6}$, convection free

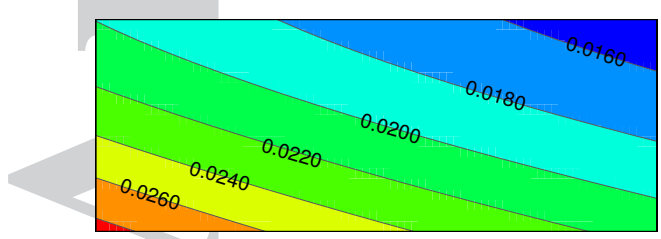

e) $C_{11}-C_{14}$, convection free

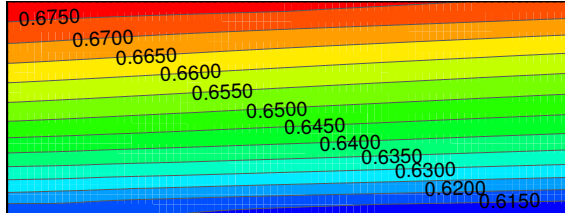

b) $C_{1}, k=10 \mathrm{md}$

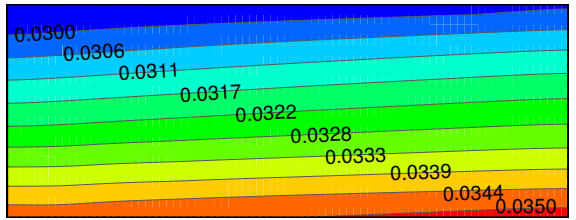

d) $C_{5}-C_{6}, k=10 \mathrm{md}$

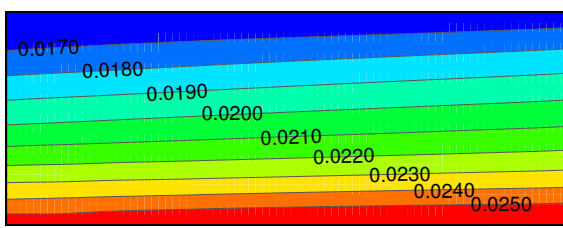

f) $C_{11}-C_{14}, k=10 \mathrm{md}$

Figure 7. Composition contour plot for $C_{1}, C_{5}-C_{6}$, and $C_{11}-C_{14}$, with different permeability cases, 10-component mixture; (Lx=10000m; Lz=1500m); Example 4b.

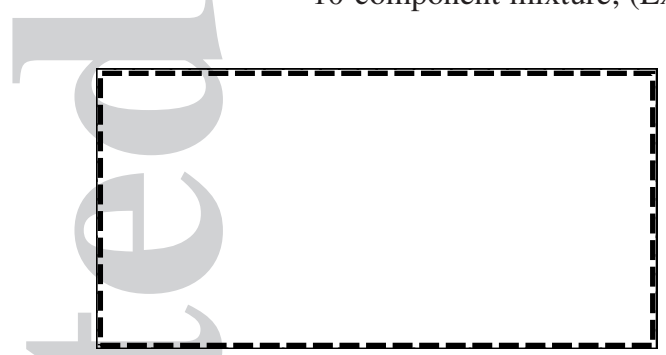

a) Configuration 1

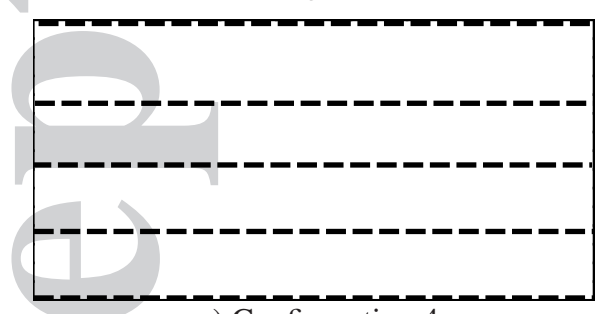

c) Configuration 4

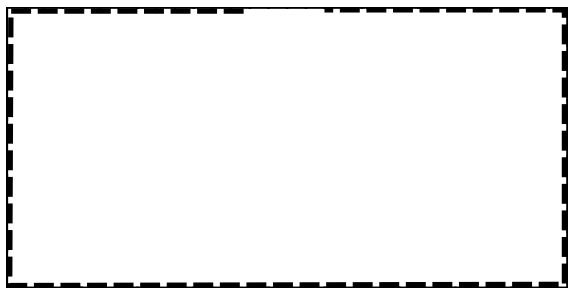

b) Configuration 2

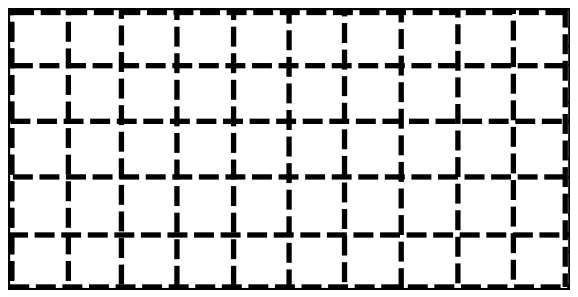

d) Configuration 4

Figure 8. Different fracture configurations; binary mixture $\left(20 \% C_{1}, 80 \% n C_{4}\right)$; Example 5 a.

cube configuration. The compositional variations and the flowline profiles for all configurations are shown in Fig. 11. In the unfractured domain (configuration 1), there is a significant horizontal variation in the methane mole fraction at the steady state (Figs. 11a, 11b). In the fractured domain (configuration 2), $C_{1}$ the compositional variation is insignificant (Figs. 11c, 11d). In configuration 3, $C_{1}$ composition contour lines are vertical in the homogeneous unfractured region and they display an " $S$ " shape in the fractured zone (Figs. 11e, 11f). The key conclusion is that the presence of fractures results in more effective mixing from natural convection. As a result, less compositional segregations from diffusion are observed. The CPU time for all these configurations, which are discretized uniformly into 1300 gridlocks, is about 700s.

Example 6: In last example, we investigate the compositional variation of a ternary mixture

This article is protected by copyright. All rights reserved. 


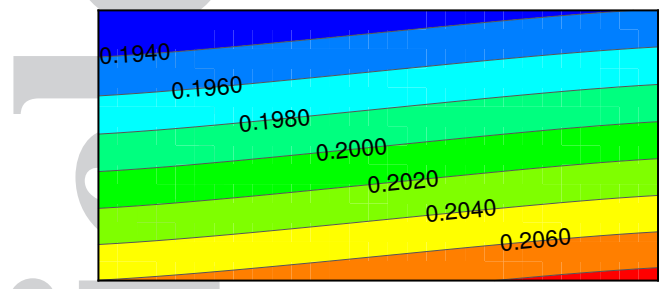

a) $k_{f}=1 m d$



b) $k_{f}=10^{3} \mathrm{md}$

Figure 9. Methane mole fraction with different fracture permeabilities; $(L x=30 \mathrm{~m} ; L z=15 \mathrm{~m})$; configuration 1, Example 5a.

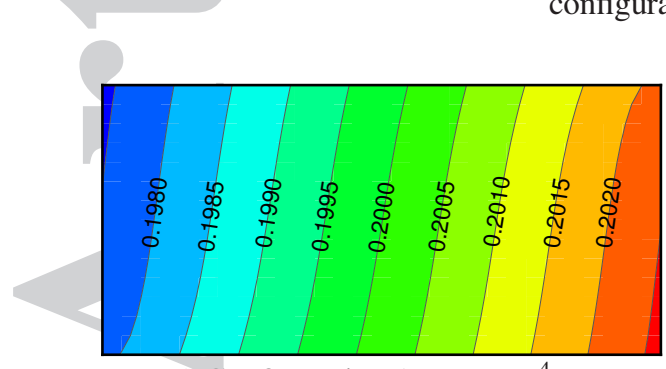

a) Configuration $1 ; k_{f}=10^{4} \mathrm{md}$

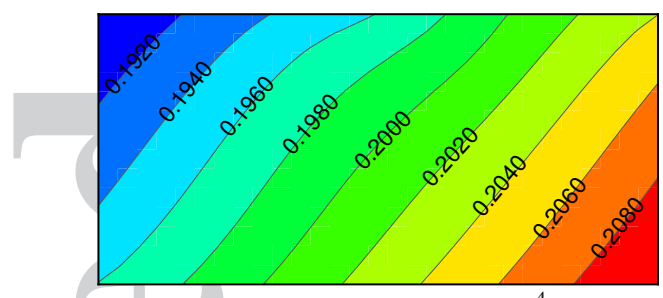

c) Configuration $2 ; k_{f}=10^{4} \mathrm{md}$

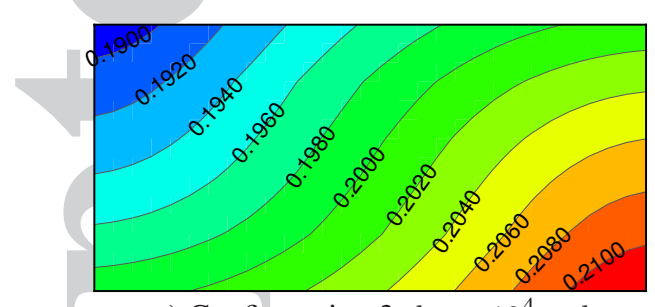

e) Configuration $3 ; k_{f}=10^{4} \mathrm{md}$

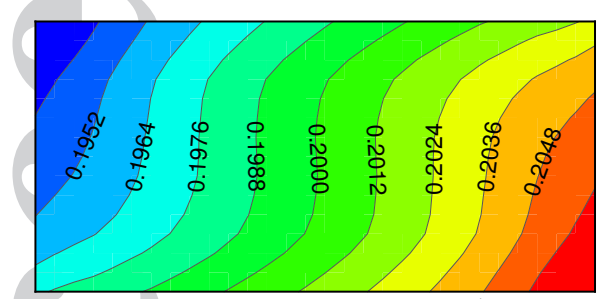

g) Configuration $4 ; k_{f}=10^{4} \mathrm{md}$

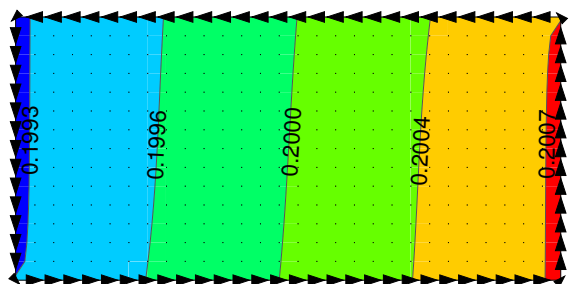

b) Configuration $1 ; k_{f}=10^{5} \mathrm{md}$

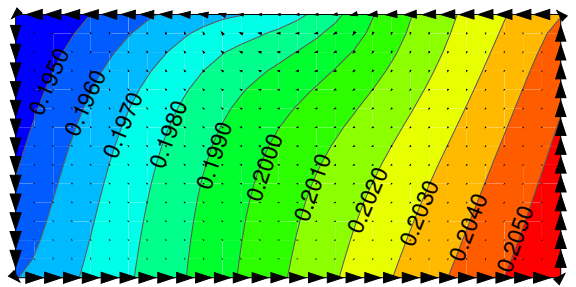

d) Configuration 2; $k_{f}=10^{5} \mathrm{md}$

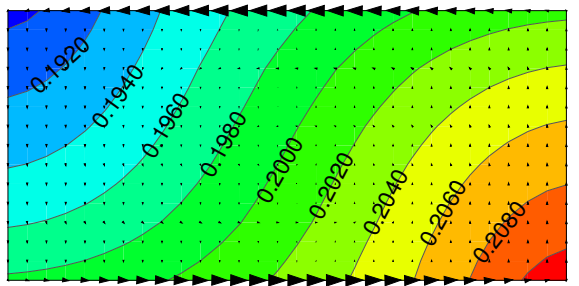

f) Configuration $3 ; k_{f}=10^{5} \mathrm{md}$

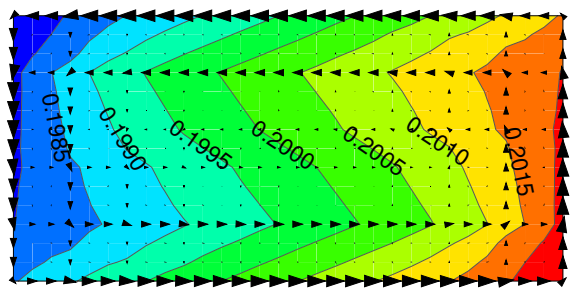

h) Configuration 4; $k_{f}=10^{5} \mathrm{md}$

Figure 10. $C_{1}$ mole fraction and velocity profiles with different fracture permeabilities and configurations, $(L x=30 m ; L z=15 m)$; Example 5a.

of $C_{1} / C_{2} / n C_{4}\left(C_{1} 35 \%, C_{2} 35 \%, n C_{4} 30 \%\right)$ in a tilted domain where different configurations for heterogeneities and fractures are considered. The relevant reservoir and fluid data are listed in Table IV. In Example 6a, we consider three configurations with different permeable zones, as shown in Fig. 12. The domain is discretized into an unstructured grid of quadrilaterals. The corresponding $C_{1}$ composition and velocity fields for all configurations are shown in Fig. 13. Different convective

This article is protected by copyright. All rights reserved. 


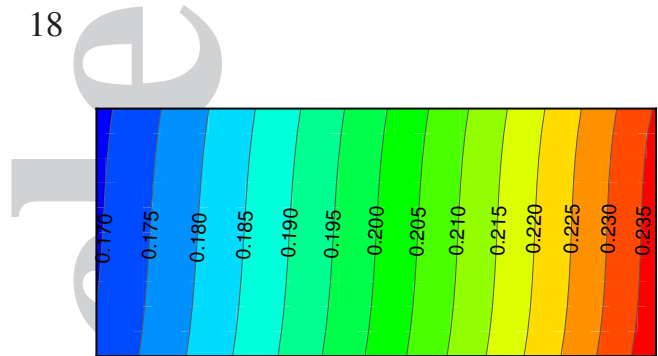

a) Unfractured; $C_{1}$ composition

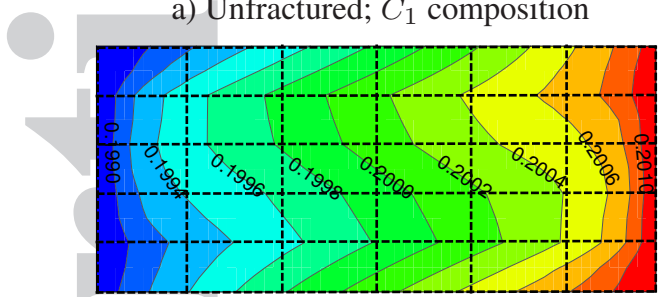

c) Sugar cube configuration; $C_{1}$ composition

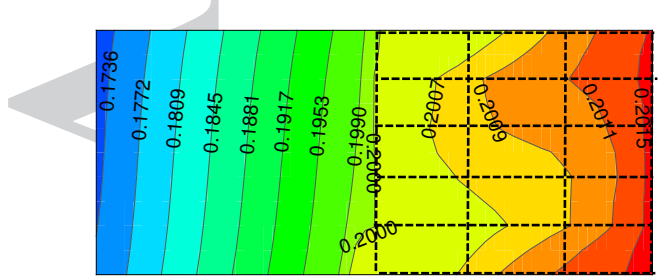

e) Partially fractured configuration; $C_{1}$ composition

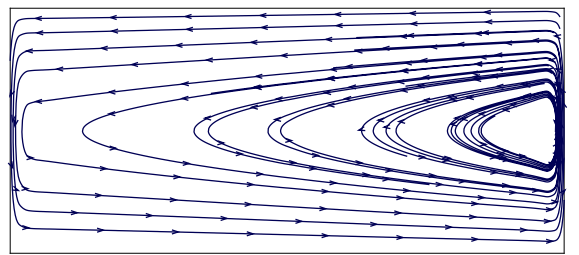

b) Unfractured; flowlines

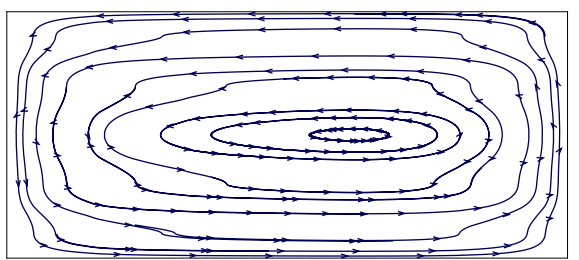

d) Sugar cube configuration; flowlines

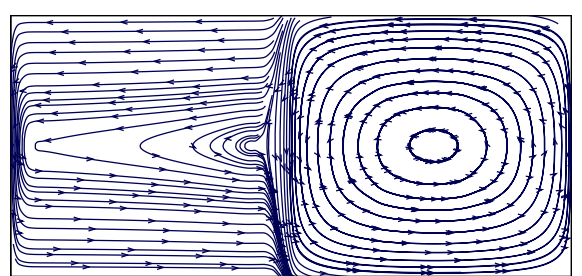

f) Partially fractured configuration; flowlines

Figure 11. $C_{1}$ mole fraction and velocity profiles with different fracture permeabilities; $(L x=6000 \mathrm{~m}$; $L z=150 \mathrm{~m})$; Example 5b.
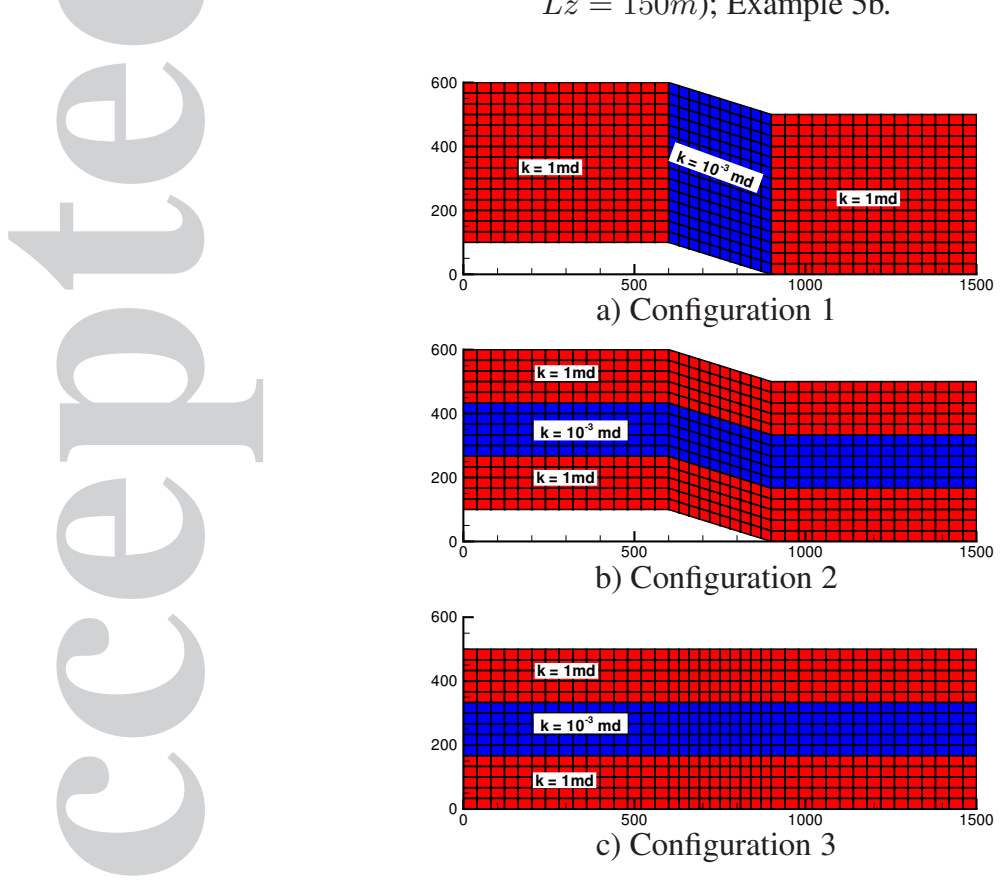

a) Configuration 1

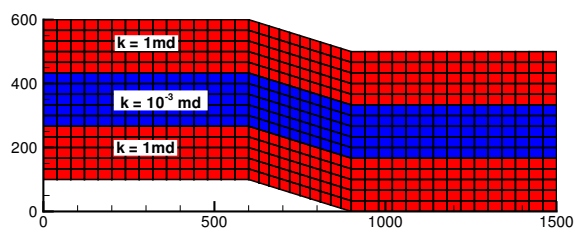

b) Configuration 2

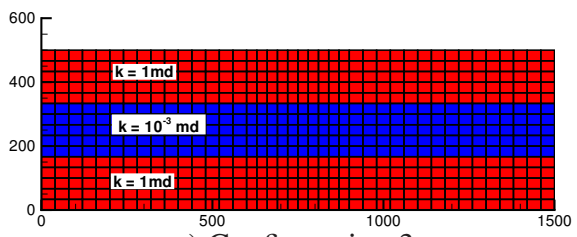

c) Configuration 3

Figure 12. Domain with different permeable zones; $(L x=1500 m, L z=600 m)$; Example 6a.

loops are observed at steady state. Due to the tilted geometry in configuration 2, the contour lines have opposite slopes in the inclined zones (Figs. 12a, 13b). The small velocity in the tight zone in configurations 2 and 3 preclude observation. In Example 6b, the domain in configurations 1 and 2 is discretized into a triangular mesh with heterogeneous permeabilities (Fig. 14). The contrasts in permeability in configurations 1 and 2 are 3 and 7 orders of magnitude, respectively. In Fig. 15,

This article is protected by copyright. All rights reserved. 


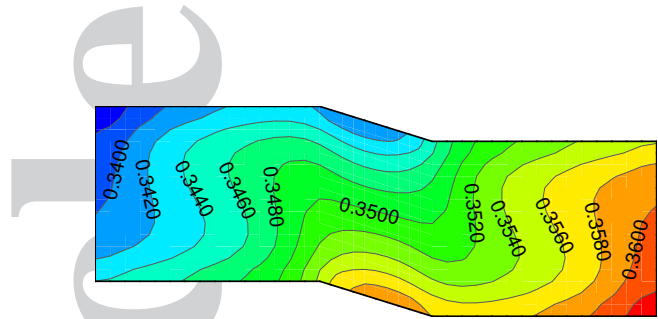

a) Configuration $1 ; C_{1}$ composition

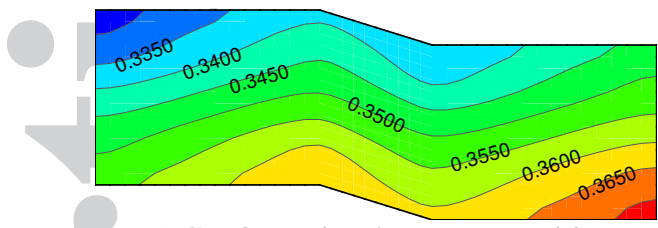

c) Configuration 2; $C_{1}$ composition

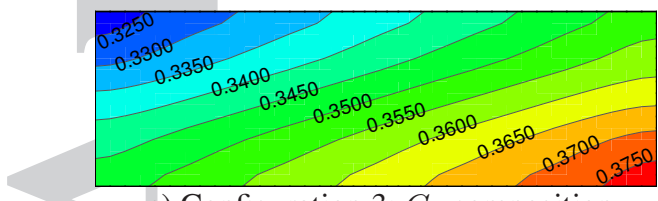

e) Configuration $3 ; C_{1}$ composition

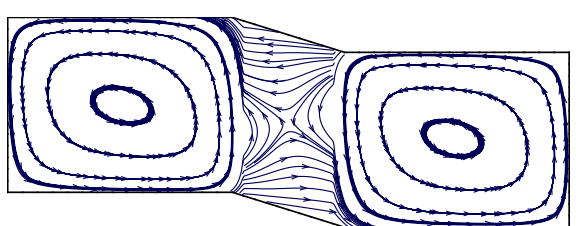

b) Configuration 1; flowlines

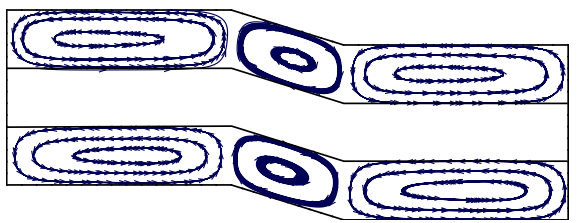

d) Configuration 2; flowlines

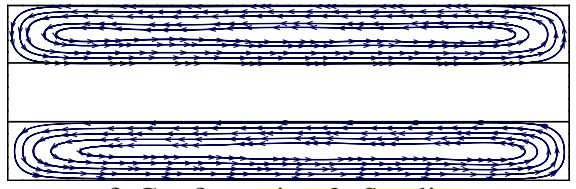

f) Configuration 3; flowlines

Figure 13. $C_{1}$ composition and velocity profiles for configurations 1-3, $(L x=1500 \mathrm{~m}, L z=600 \mathrm{~m})$; Example 6a.

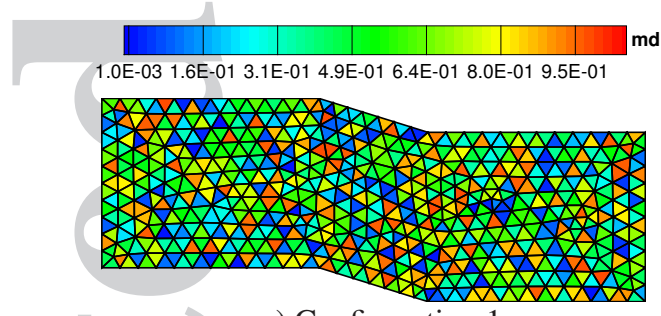

a) Configuration 1

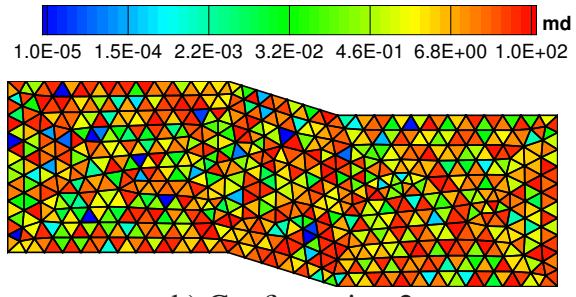

b) Configuration 2

Figure 14. Permeability distributions for two configurations, $(L x=1500 m, L z=600 m)$; Example $6 b$.

we compare the contour lines with the case where the domain is assumed homogeneous with a permeability $k=0.1 \mathrm{md}$. The plots show that, in all cases, there is only one main convection cell. In Example 6c, we consider three configurations of connected and disconnected fractures (Fig. 16). The contour lines for $C_{1}$ composition and the velocity fields are represented in Fig. 17. The results are similar to those for the rectangular domain with structured fractures. However, from a numerical perspective, this highlights the flexibility of our numerical algorithm to model unstructured domains, including those with connected and disconnected fractures.

\section{SUMMARY AND CONCLUSIONS}

An efficient and robust numerical approach using the DG and MFE methods is presented for computing compositional variation of multicomponent mixtures in homogeneous, heterogeneous, and fractured porous media. With this numerical approach, we solve the unsteady state convection/diffusion equations without adopting the Oberbeck-Boussinesq approximation. The diffusion coefficients and density are considered variables in space and time. We use the DG method to approximate the flow equations, which produces less numerical dispersion than the conventional upwind FD method. The MFE method is used to approximate the pressure unknown and the convective fluxes (velocity field). From our numerical experiments, we conclude that the MFE method in computing the convection fluxes has a high accuracy for systems having high contrast 




a) Homogeneous; $C_{1}$ composition

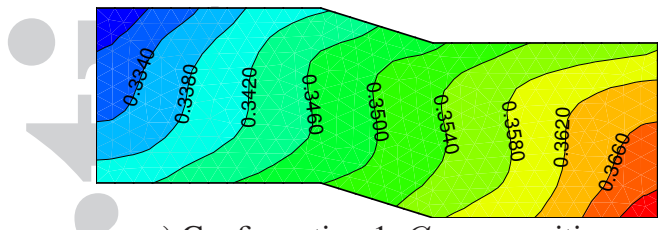

c) Configuration $1 ; C_{1}$ composition

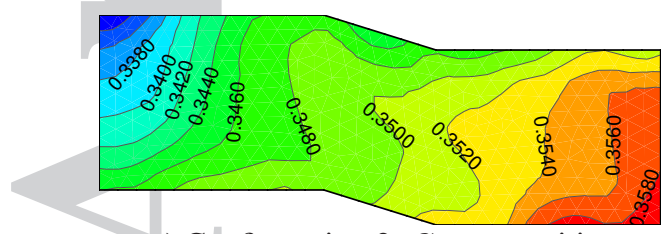

e) Configuration 2; $C_{1}$ composition

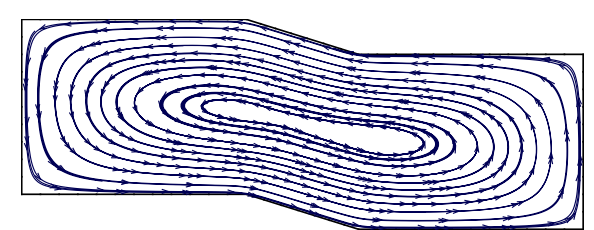

b) Homogeneous; flowlines

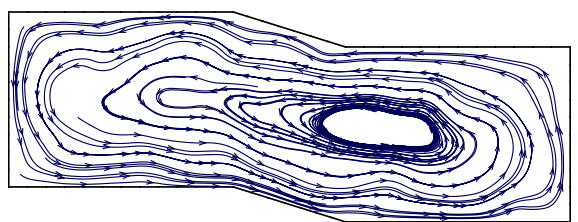

d) Configuration 1; flowlines

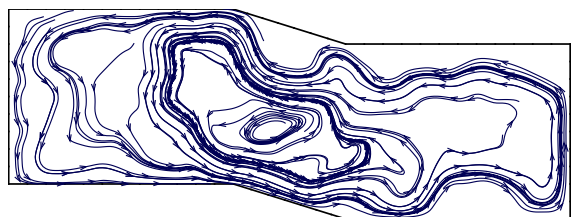

f) Configuration 2; flowlines

Figure 15. $C_{1}$ composition and velocity profiles for configurations 1-2, $(L x=1500 m, L z=600 m)$; Example 6b.
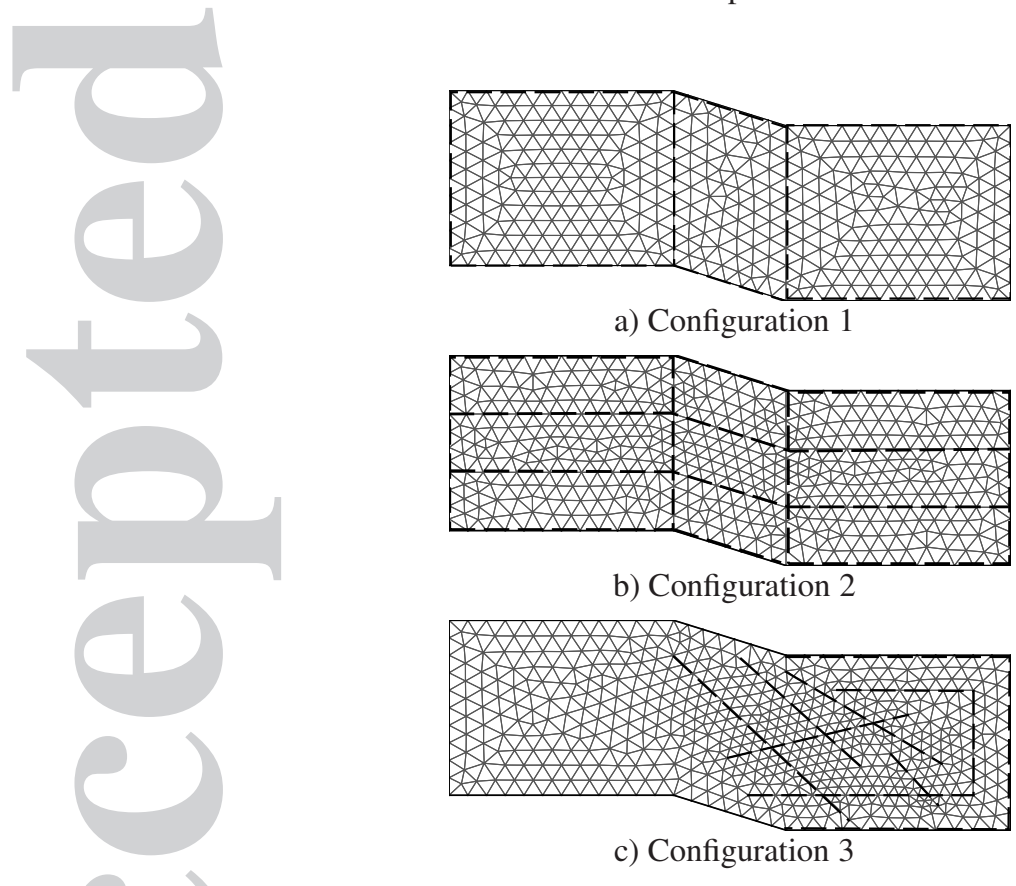

a) Configuration 1

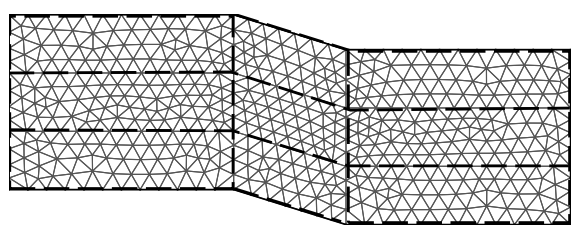

b) Configuration 2

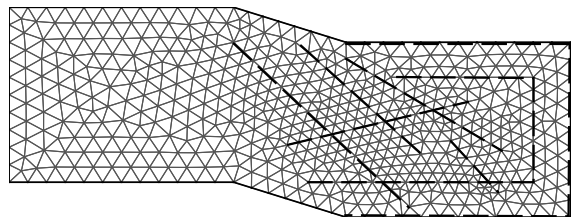

c) Configuration 3

Figure 16. Models with different fractured configurations; $(L x=1500 m, L z=600 m)$; Example 6c.

in permeability $\left(10^{7}\right)$ or non-smooth gridding. In contrast, the two-point FD and FV approximations are not accurate enough to approximate the velocity field on unstructured grids, even if the mesh is smooth.

To approximate the molecular diffusion fluxes, an alternative approach to the MFE method based on the LDG concept is implemented. The MFE and LDG methods are similar on the fact that both approximate the main unknown and its gradient simultaneously. The main difference between them is that the MFE method leads to a global system to calculate the fluxes, whereas, in LDG method, the

This article is protected by copyright. All rights reserved. 


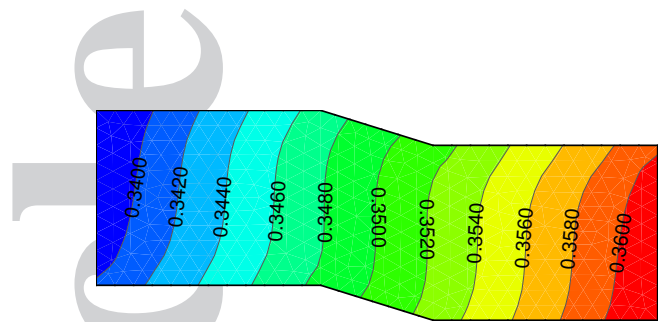

a) Configuration $1 ; C_{1}$ composition

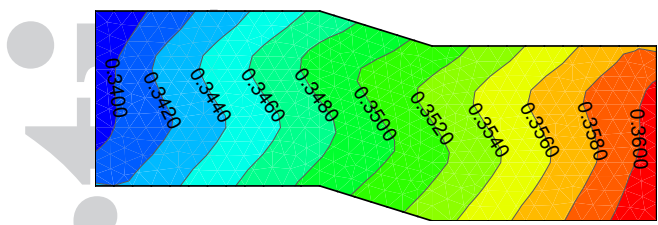

c) Configuration 2; $C_{1}$ composition

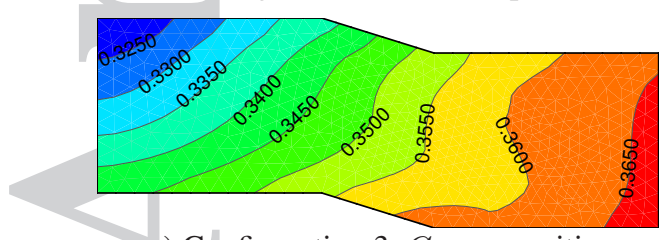

e) Configuration $3 ; C_{1}$ composition

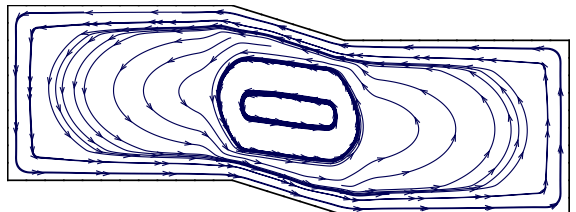

b) Configuration 1; flowlines

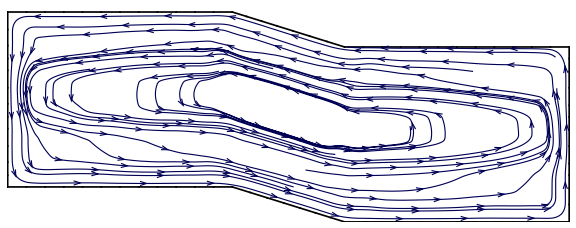

d) Configuration 2; flowlines

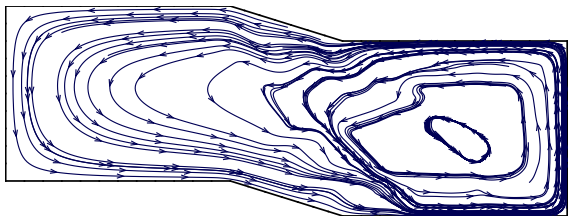

f) Configuration 3; flowlines

Figure 17. $C_{1}$ composition and velocity profiles for configurations 1-3, $(L x=1500 \mathrm{~m}, L z=600 \mathrm{~m})$; Example 6c.

fluxes are calculated locally. Therefore, the LDG method is less costly from a numerical perspective. However, numerical comparisons show that the MFE is superior, especially for non-smooth grids.

For fractured media, the discrete fracture model is implemented so that the fractures are treated as $1 \mathrm{D}$ entities. This approach reduces dramatically the CPU time since it avoids the complexities of the single-porosity model (e.g., sharp spatial variations of parameters in matrix and fractures, refined gridding due to the length-scale difference between matrix and fractures).

\section{APPENDIX}

\section{A - MODELING FRACTURES}

A key step in the discrete fracture modeling is to represent 2D fracture cells with $1 \mathrm{D}$ elements, as shown in Fig. A1. From the crossflow equilibrium concept [19, 20, 21, 45, 48, 49], the pressure in a fracture cell and the pressures in the neighboring matrix cells are assumed equal. By integrating the governing equations over the control volume that includes the fracture and the corresponding matrix cells alleviates the computation of the matrix-fracture fluxes. This technique is accurate and more efficient than the single-porosity.

Let $K$ be a grid block that contains a 1D fracture element $I$. By applying the mixed hybrid finite element formulation to Darcy's law over K, the flux $q_{K, E}$ across the edge $E$ of $K$ can be expressed as a function of the cell pressure average $p_{K}$ and the edge pressure averages (pressure traces) $t p_{K, E}$, as shown in Eq. 11. The same approach can be applied to discretized Darcy's law in the fracture element I. The flux $q_{I, e}$ across the extremity $e$ of $I$ can then be written as:

$$
q_{I, e}=\alpha_{I, e} p_{I}-\sum_{e^{\prime} \in \partial I} \beta_{I, e, e^{\prime}} t p_{I, e^{\prime}}-\gamma_{I, e} \quad e \in \partial I .
$$

where $p_{I}$ and $t p_{I, e}$ are respectively the pressure average on $I$ and the pressure traces at the extremities of $I$. In the grid cells that contain fractures, the block pressure average and the fracture slice average are equal, that is, $p_{K}=p_{I}$. By imposing the continuity of the fluxes, the flux unknown can then be eliminated and a linear system with the pressure average $P$ and the pressure trace $T_{P}$ is obtained, as in Eq. 12. 


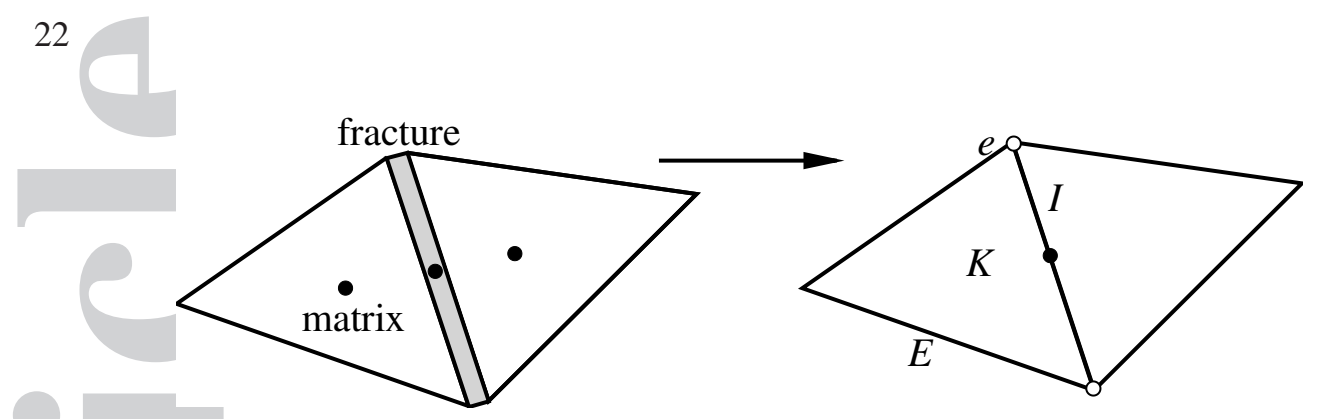

Figure A1. Geometric simplification of 2D fracture cells into 1D fracture cells.

The second step is to discretize the material balance equations. The integration of the 2 nd flow equation in 6 over the matrix and fracture elements gives:

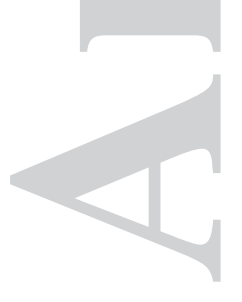

$$
\begin{gathered}
\left(\int_{K} \phi \frac{\partial \varsigma_{K, i}}{\partial t}+\int_{I} \phi \frac{\partial \varsigma_{I, i}}{\partial t}\right)+\left(\int_{K} \varsigma_{K, i} \boldsymbol{\vartheta}+\int_{I} \varsigma_{I, i} \boldsymbol{\vartheta}\right) \\
+\left(\int_{K} \nabla . \boldsymbol{J}_{i}+\int_{I} \nabla \cdot \boldsymbol{J}_{i}\right)=0, \quad i=1, \ldots, n_{c}-1 .
\end{gathered}
$$

Using the divergence theorem (see Eq. 8 ) and assuming that the average concentrations in the matrix and fracture cells are equal, Eq.A2 simplifies to:

$$
\begin{aligned}
& \left(\phi_{K}|K|+|I| \phi_{I}\right) \frac{d \bar{\varsigma}_{i, K}}{d t}+\sum_{E \subset \partial K} q_{K, E} \tilde{\varsigma}_{K, E}+\varepsilon \sum_{e \subset \partial I} q_{I, e} \tilde{\varsigma}_{I, e} \\
& +\sum_{\partial K} f_{i}^{T, p, M}+\varepsilon \sum_{\partial I} f_{i}^{T, p, M}=0 .
\end{aligned}
$$

where $|K|$ and $|I|$ are respectively the volumes of matrix and fracture cells; $\varepsilon$ is the fracture aperture; $\bar{\varsigma}_{i, K}$ the cell averages of $\varsigma_{i, K}$, respectively; $\tilde{\varsigma}_{K, E}$ and $\tilde{\varsigma}_{I, e}$ are the concentrations from the upstream matrix and fracture cells, and $f_{i}^{T, p, M}$ represent the thermal, pressure, and molecular diffusion fluxes in the matrix and fractures. The expressions of these fluxes are given in Eqs. 18, 19, and 24. Note the integration of the first equation in 6 can be done similarly but without the diffusion term.

\section{REFERENCES}

1. Oliveira Padua KG. Oil Composition Variation in a Large Deep Water Field. In: SPE Latin American and Caribbean Petroleum Engineering Conference; 1997; Rio de Janeiro, Brazil.

2. D Gupta, Lawrence J Naguib Bin Ab Majid M, T Wahlheim. Fluid Characterization and Modeling of Compositional Variation, Dukhan Field. In: SPE Conference: International Petroleum Technology Conference; 2009.

3. Nasrabadi H, Firoozabadi A, Esposito, Jaime Mello Vieira A. Interpretation of an Unusual Bubblepoint Pressure Variation in an Offshore Field. In: SPE Conference: Europec/EAGE Conference and Exhibition; 2008.

4. Metcalfe RS, Vogel JL, Morris RW. Compositional Gradients in the Anschutz Ranch East Field. SPE Journal. ;3(3):1025-1032.

5. Hamoodi AN, Abed A, J Grabenstetter. Modeling of a Large Gas-Capped Reservoir with Areal and Vertical Variation in Composition. In: SPE Conference: 69th Annual SPE Technical Conference and Exhibition; 1994.

6. Lage JL, Nield DA. Convection induced by inclined gradients in a shallow porous medium layer. Journal of Porous Media. 1998;1(1):57-69.

7. Thomas O. Reservoir Analysis based on compositional gradients. Stanford University: PhD thesis; 2007.

8. Nikpoor M. Modeling of Compositional Grading in Nonisothermal Petroleum Reservoirs. University of Calgary: $\mathrm{PhD}$ thesis; 2014.

9. Ratulowski J, Fuex AN, Westrich JT, Sieler JJ. Theoretical and Experimental Investigation of Isothermal Compositional Grading. SPE Reservoir Evaluation and Engineering. ;6(3):168-175.

10. PL Montel, Gouel . Prediction of Compositional Grading in a Reservoir Fluid Column. In: SPE Conference: SPE Annual Technical Conference and Exhibition; 1985.

11. Bolton EW, Firoozabadi A. Numerical modeling of temperature and SPEcies distributions in hydrocarbon reservoirs. Journal of Geophysical Research-Solid Earth. 2014;119(1):18-31.

This article is protected by copyright. All rights reserved. 
12. Whitson C, Belery P. Compositional Gradients in Petroleum Reservoirs. In: University of Tulsa Centennial Petroleum Engineering Symposium; 1994.

13. Hier Lars, Whitson CH. Compositional GradingTheory and Practice. SPE Journal. 2001;:525-535.

14. Jacqmin D. Interaction of Natural Convection and Gravity Segregation in Oil/Gas Reservoirs. SPE Journal. ;:233.

15. Riley MF, Firoozabadi A. Compositional variation in hydrocarbon reservoirs with natural convection and diffusion. Aiche Journal. 1998;44(2):452-464.

16. Ghorayeb K, Firoozabadi A. Modeling multicomponent diffusion and convection in porous media. SPE Journal. 2000;5(2):158-171.

17. Ghorayeb K, Firoozabadi A. Features of convection and diffusion in porous media for binary systems. Journal of Canadian Petroleum Technology. 2001;40(2):21-28.

18. Ghorayeb K, Firoozabadi A. Numerical study of natural convection and diffusion in fractured porous medics. SPE Journal. 2000;5(1):12-20.

19. Hoteit H, Firoozabadi A. Multicomponent fluid flow by discontinuous Galerkin and mixed methods in unfractured and fractured media. Water Resources Research. 2005;41(11).

20. Hoteit H, Firoozabadi A. Compositional modeling of discrete-fractured media without transfer functions by the discontinuous Galerkin and mixed methods. SPE Journal. 2006;11(3):341-352.

21. Hoteit H, Firoozabadi A. Numerical Modeling of Diffusion in Fractured Media for Gas-Injection and -Recycling Schemes. SPE Journal. 2009;14(2):323-337.

22. T Russell, Wheeler MF. Finite element and finite difference methods for continuous flows in porous media. SIAM, The Mathematics of Reservoir Simulation. 1983;:35-106.

23. Chavent G, Roberts JE. A Unified Physical Presentation of Mixed, Mixed-Hybrid Finite-Elements and Standard Finite-Difference Approximations for the Determination of Velocities in Waterflow Problems. Advances in Water Resources. 1991;14(6):329-348.

24. Weiser A, Wheeler MF. On Convergence of Block-Centered Finite-Differences for Elliptic Problems. SIAM Journal on Numerical Analysis. 1988;25(2):351-375.

25. Arbogast T, Dawson CN, Keenan PT, Wheeler MF, Yotov I. Enhanced cell-centered finite differences for elliptic equations on general geometry. SIAM Journal on Scientific Computing. 1998;19(2):404-425.

26. Arbogast T, Wheeler MF, Yotov I. Mixed finite elements for elliptic problems with tensor coefficients as cellcentered finite differences. SIAM Journal on Numerical Analysis. 1997;34(2):828-852.

27. Wheeler M, Xue G R, Yotov I. A multipoint flux mixed finite element method on distorted quadrilaterals and hexahedra. Numerische Mathematik. 2012;121(1):165-204.

28. Wheeler MF, Yotov I. A multipoint flux mixed finite element method. SIAM Journal on Numerical Analysis. 2006;44(5):2082-2106.

29. Durlofsky LJ. Accuracy of Mixed and Control-Volume Finite-Element Approximations to Darcy Velocity and Related Quantities. Water Resources Research. 1994;30(4):965-973.

30. Mose R, Siegel P, Ackerer P, Chavent G. Application of the Mixed Hybrid Finite-Element Approximation in a Groundwater-Flow Model - Luxury or Necessity. Water Resources Research. 1994;30(11):3001-3012.

31. Raviart P, Thomas J. A mixed hybrid finite element method for the second order elliptic problem. New York: Springer-Verlag; 1977.

32. Brezzi F, Fortin M. Mixed and Hybrid Finite Element Method. New York: Springer-Verlag; 1991.

33. Yotov I. Mixed Finite Element Methods for Flow in Porous Media. University of Texas: PhD thesis; 1996.

34. Chavent G, Jaffre J. Mathematical models and finite elements for reservoir simulation. North-Holland: Elsevier; 1986.

35. Sun SY, Wheeler MF. Discontinuous Galerkin methods for coupled flow and reactive transport problems. Applied Numerical Mathematics. 2005;52(2-3):273-298.

36. Girault V, Sun SY, Wheeler MF, Yotov I. Coupling discontinuous Galerkin and mixed finite element discretizations using mortar finite elements. SIAM Journal on Numerical Analysis. 2008;46(2):949-979.

37. Kim M, Wheeler MF. Coupling discontinuous Galerkin discretizations using mortar finite elements for advectiondiffusion-reaction problems. Computers and Mathematics with Applications. 2014;67:181-198.

38. Cockburn B, Shu CW. The local discontinuous Galerkin method for time-dependent convection-diffusion systems. SIAM Journal on Numerical Analysis. 1998;35(6):2440-2463.

39. Karimi-Fard M, Firoozabadi A. Numerical simulation of water injection in fractured media using the discretefracture model and the Galerkin method. SPE Reservoir Evaluation and Engineering. 2003;6(2):117-126.

40. Karimi-Fard M, Durlofsky LJ, Aziz K. An efficient discrete-fracture model applicable for general-purpose reservoir simulators. SPE Journal. 2004;9(2):227-236.

41. Peng D, Robinson DB. A New Two-Constant Equation of State. Industrial and Engineering Chemistry Fundamentals. 1976;15(1):59-64.

42. Firoozabadi Abbas. Thermodynamics of hydrocarbon reservoirs. New York: McGraw-Hill; 1999.

43. Ghorayeb K, Firoozabadi A. Molecular, pressure, and thermal diffusion in nonideal multicomponent mixtures. Aiche Journal. 2000;46(5):883-891.

44. Firoozabadi A, Ghorayeb K, Shukla K. Theoretical model of thermal diffusion factors in multicomponent mixtures. Aiche Journal. 2000;46(5):892-900.

45. Hoteit H, Firoozabadi A. Compositional modeling by the combined discontinuous Galerkin and mixed methods. SPE Journal. 2006;11(1):19-34.

46. Hoteit H, Ackerer P, Mose R, Erhel J, Philippe B. New two-dimensional slope limiters for discontinuous Galerkin methods on arbitrary meshes. International Journal for Numerical Methods in Engineering. 2004;61(14):25662593.

47. Fagherazzi S, Furbish DJ, Rasetarinera P, Hussaini MY. Application of the discontinuous SPEctral Galerkin method to groundwater flow. Advances in Water Resources. 2004;27(2):129-140.

48. Noorishad J, Mehran M. An Upstream Finite-Element Method for Solution of Transient Transport-Equation in Fractured Porous-Media. Water Resources Research. 1982;18(3):588-596. 
49. Baca RG, Arnett RC, Langford DW. Modeling Fluid-Flow in Fractured Porous Rock Masses by Finite-Element Techniques. International Journal for Numerical Methods in Fluids. 1984;4(4):337-348.
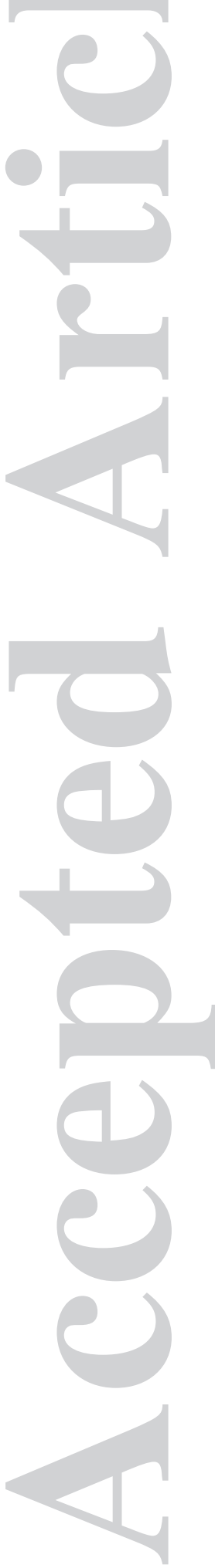

This article is protected by copyright. All rights reserved. 